\title{
Probing deconfinement in a chiral effective model with Polyakov loop at imaginary chemical potential
}

\section{$\operatorname{AUTHOR}(S)$ :}

Morita, Kenji; Skokov, Vladimir; Friman, Bengt; Redlich, Krzysztof

\section{CITATION:}

Morita, Kenji ... [et al]. Probing deconfinement in a chiral effective model with Polyakov loop at imaginary chemical potential. Physical Review D 2011, 84(7): 076009.

\section{ISSUE DATE:}

2011-10

URL:

http://hdl.handle.net/2433/152374

RIGHT:

(C) 2011 American Physical Society 
PHYSICAL REVIEW D 84, 076009 (2011)

\title{
Probing deconfinement in a chiral effective model with Polyakov loop at imaginary chemical potential
}

\author{
Kenji Morita, ${ }^{1,2, *}$ Vladimir Skokov, ${ }^{1}$ Bengt Friman, ${ }^{1}$ and Krzysztof Redlich ${ }^{3,4}$ \\ ${ }^{1}$ GSI, Helmholzzentrum für Schwerionenforschung, Planckstr. 1, D-64291 Darmstadt, Germany \\ ${ }^{2}$ Yukawa Institute for Theoretical Physics, Kyoto University, Kyoto 606-8502, Japan \\ ${ }^{3}$ Institute of Theoretical Physics, University of Wroclaw, PL-50204 Wroctaw, Poland \\ ${ }^{4}$ Extreme Matter Institute EMMI, GSI, Planckstr. 1, D-64291 Darmstadt, Germany
}

(Received 12 July 2011; published 28 October 2011)

\begin{abstract}
The phase structure of the two-flavor Polyakov-loop extended Nambu-Jona-Lashinio model is explored at finite temperature and imaginary chemical potential with a particular emphasis on the confinementdeconfinement transition. We point out that the confined phase is characterized by a $\cos 3 \mu_{I} / T$ dependence of the chiral condensate on the imaginary chemical potential while in the deconfined phase this dependence is given by $\cos \mu_{I} / T$ and accompanied by a cusp structure induced by the $Z(3)$ transition. We demonstrate that the phase structure of the model strongly depends on the choice of the Polyakov loop potential $\mathcal{U}$. Furthermore, we find that by changing the four fermion coupling constant $G_{s}$, the location of the critical end point of the deconfinement transition can be moved into the real chemical potential region. We propose a new parameter characterizing the confinement-deconfinement transition.
\end{abstract}

DOI: 10.1103/PhysRevD.84.076009

\section{INTRODUCTION}

The exploration of the phase diagram of strongly interacting matter has received a lot of attention in recent years. First principle calculations of the phase structure from the Lagrangian of quantum chromodynamics (QCD) is intrinsically difficult owing to the strongly coupled nature of the theory at large distances. Lattice gauge theory (LGT) calculations provide a unique and powerful tool for studying QCD in the nonperturbative regime. Increasing computer power has recently made LGT simulations at almost physical quark masses possible $[1,2]$. The results indicate that the transition from the confined, chirally broken phase to the deconfined, chirally restored phase at $T \sim 160 \mathrm{MeV}$ and vanishing baryon chemical potential, $\mu_{B}=0$, is of the crossover type [3].

For nonzero net baryon density, LGT calculations suffer from the so-called "sign problem." For finite quark chemical potential (and $N_{c}=3$ ), the statistical weight of the Monte Carlo simulation becomes nonpositive definite due to the complex fermion determinant. This issue has impeded the progress in LGT calculations at finite densities.

There have been several attempts to bypass the sign problem [4]. One interesting approach involves using an imaginary chemical potential, for which the fermion determinant is real and, therefore, systematic LGT simulations are possible [5]. There are two major ways for extracting information on the real phase diagram from calculations at imaginary chemical potential. One is to project the grand partition function $Z_{G}$ computed at imaginary chemical potential onto the canonical partition function

*kmorita@yukawa.kyoto-u.ac.jp
PACS numbers: 11.30.Rd, 12.38.Aw, 12.39.Fe, $25.75 . \mathrm{Nq}$

$$
Z_{c}\left(T, V, N_{q}\right)=\int_{0}^{2 \pi} \frac{d\left(\beta \mu_{I}\right)}{2 \pi} e^{-i \beta \mu_{I} N_{q}} Z_{G}\left(T, V, \mu_{q}=i \mu_{I}\right)
$$

In spite of the difficulties involved in the evaluation of the oscillatory integral, there are lattice calculations aimed at studying the phase diagram in the temperature-number density plane by means of this approach [6,7]. An alternative way involves an analytic continuation from imaginary to real values of the chemical potential. This method has proven quite powerful for determining the critical line at $\mu_{q}<\pi T / 3$ [8] and this approach has been applied in LGT calculations [9-18], in resummed perturbation theory [19], as well as in quasiparticle models [20]. Of course, the analytic continuation requires knowledge of the analytic structure of the thermodynamic functions. Therefore effective models that share the symmetries of QCD are useful for testing such approaches, because a result obtained by analytic continuation from imaginary chemical potential can be confronted with the known solution at real chemical potential.

A remarkable feature of QCD at imaginary chemical potential is the Roberge-Weiss (RW) transition at $\mu_{q} / T=$ $\pi / 3+2 \pi k / 3$, where $k$ is an integer [5]. The RW transition involves a shift from one $Z(3)$ sector to another in the deconfined phase. This transition is a remnant of the $Z(3)$ symmetry of the pure gauge theory, which is explicitly broken in the presence of fermions of finite mass. Note that in this case the Polyakov loop is not an exact order parameter. Using perturbation theory [5], Roberge and Weiss showed that this phase transition is first-order. This was confirmed in subsequent lattice simulations [8]. While it was expected that the RW transition is a signature of the deconfined phase [21], the transition line, which is parallel 
to the temperature axis at $\mu_{I} / T=\pi / 3$, terminates at a temperature above the deconfinement transition temperature at vanishing chemical potential. Since the characteristics of the end point and its implications for the phase diagram at real $\mu$ are still debated [16,17,22-26], it is interesting to explore the phase structure at imaginary chemical potential in an effective model. The aim of this paper is to characterize the phase structure especially of the confinement-deconfinement transition of QCD at imaginary chemical potential in the framework of an effective model which exhibits the relevant symmetries.

In this work, we use the Polyakov-loop-extended Nambu-Jona-Lasinio (PNJL) model [27,28]. The NJL model $[29,30]$ describes many aspects of QCD related to chiral symmetry [31]. This model, however, lacks confinement. On the other hand, thermal models with internal gauge symmetry have been studied. These models reveal a RW transition [32,33] while chiral symmetry is not realized. The PNJL model is an effective model of QCD, which ameliorates some of the shortcomings of the NJL model by introducing a coupling of the quark field to a uniform background gauge field $A_{0}$. It has been demonstrated that the PNJL model reproduces the RW transition [34]. The authors of Ref. [34] have studied the phase structure of the PNJL model in detail (see also $[22,24,35-39])$. These studies indicate that various improvements are necessary in order to reproduce the lattice data. In this paper, applying the simplest interaction term in the PNJL model as introduced in Ref. [34], we focus on differences in the behavior of the order parameters dependent on the parametrizations of the effective Polyakov-loop potential. We characterize the phase structure qualitatively through a systematic comparison of the results for different Polyakov-loop potentials and give perspectives on the nature of the phase transitions at imaginary chemical potential.

We introduce a new quantity, which characterizes the confinement-deconfinement transition based on the characteristic dependence of the chiral condensate on the imaginary chemical potential. The relation of this parameter to the so-called dual order parameter [40] is discussed.

The paper is organized as follows: in the next section, we briefly review the basic properties of the QCD partition function which are relevant for this study. The model is introduced in Sec. III and results of the numerical calculation are presented in Sec. IV. In Sec. V we discuss the parameters characterizing the confinement-deconfinement transition, and finally in Sec. VI we summarize.

\section{GENERAL PROPERTIES OF THE QCD PARTITION FUNCTION AT IMAGINARY CHEMICAL POTENTIAL}

The partition function of the $S U\left(N_{c}\right)$ gauge theory with fermions, is characterized by the number operator $\hat{N}=$ $\int d^{3} x q^{\dagger} q$, at imaginary chemical potential $\theta=\mu_{I} / T=\beta \mu_{I}$.

$$
Z_{G}(T, V, \theta)=\operatorname{Tr}\left[e^{-\beta H+i \theta \hat{N}}\right] .
$$

The partition function can be expressed in terms of the functional integral

$$
Z_{G}(T, V . \theta)=\int \mathcal{D} q \mathcal{D} \bar{q} \mathcal{D} A_{\mu} e^{-S_{E}(\beta, \theta)}
$$

where $S_{E}$ is the Euclidean action

$$
S_{E}=\int_{0}^{\beta} d \tau \int d^{3} x \bar{q}(\gamma \cdot D-m) q-\frac{1}{4} G^{2}-i \frac{\theta}{\beta} q^{\dagger} q .
$$

In (3), the gauge and quark field obey periodic and antiperiodic boundary conditions in the temporal interval $[0, \beta]$, respectively. The particle-antiparticle symmetry implies, that $Z_{G}$ is an even function of $\theta, Z_{G}(T, V,-\theta)=$ $Z_{G}(T, V, \theta)$.

By performing the change of variables $q \rightarrow e^{i \theta \tau / \beta} q$, the explicit dependence on imaginary chemical potential in the action can be removed and converted into a modified boundary condition $[5,21]$

$$
q(x, \beta)=-e^{i \theta} q(x, 0) .
$$

Then the $Z\left(N_{c}\right)$ transformation

$$
q \rightarrow U q, \quad A_{\mu} \rightarrow U A_{\mu} U^{-1}-(i / g)\left(\partial_{\mu} U\right) U^{-1}
$$

with $U(x, \beta)=\exp \left(2 \pi i k / N_{c}\right) U(x, 0)$ leaves the action and the functional measure invariant, but modifies the boundary condition

$$
q(x, \beta)=-e^{i \theta} e^{\left(2 \pi i / N_{c}\right) k} q(x, 0),
$$

where $k$ is an integer [5,21]. A comparison of Eqs. (7) and (5) reveals that the partition function is periodic with respect to finite shifts of $\theta$

$$
Z_{G}(T, V, \theta)=Z_{G}\left(T, V, \theta+\frac{2 \pi k}{N_{c}}\right) .
$$

This periodicity is a remnant of the $Z\left(N_{c}\right)$ symmetry, the center symmetry of the $S U\left(N_{c}\right)$ gauge group. In the presence of fermions, the $Z\left(N_{c}\right)$ symmetry is explicitly broken. In other words, the partition function is invariant under the transformation $\theta \rightarrow \theta+2 \pi k / N_{c}$ combined with the $Z\left(N_{c}\right)$ transformation. This symmetry was dubbed "extended $Z\left(N_{c}\right)$ symmetry" in Ref. [34]. It is easy to see that the thermodynamic potential $\Omega=-T \ln Z_{G}$ and the chiral condensate $\sigma \equiv\langle\bar{q} q\rangle=-\frac{1}{V} \frac{\partial \Omega}{\partial m_{0}}$, with $m_{0}$ being the current quark mass, have the same periodicity.

Roberge and Weiss noted the existence of the first-order transition at $\theta=\pi / N_{c}+2 \pi k / N_{c}$ in the deconfined phase [5]. It was expected that the RW transition takes place at the same temperature as the confinement-deconfinement transition temperature $T_{d}$. Lattice simulations, however, showed that the end point of the RW transition is at a temperature $T_{E}$, which is higher than $T_{d}$. A schematic phase diagram for imaginary chemical potential is shown in Fig. 1. The nature of the chiral and confinement-deconfinement 


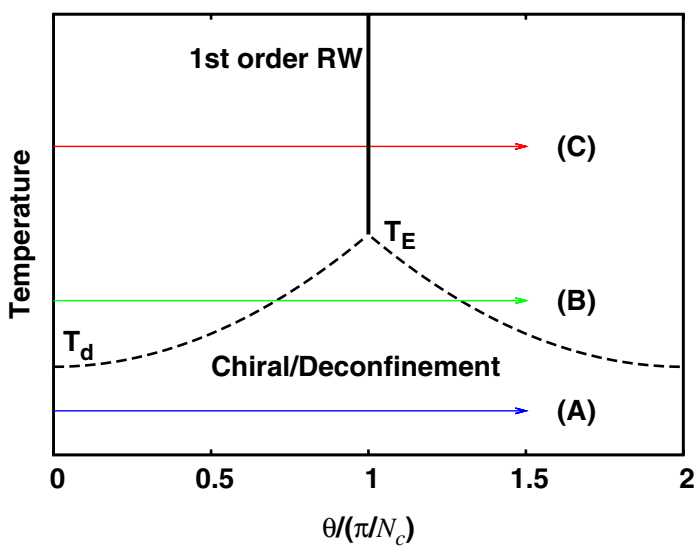

FIG. 1 (color online). Schematic phase diagram on $T-\theta$ plane. The solid line denotes the first-order Roberge-Weiss transition line which terminates at $T=T_{E}$, while the dashed line shows the transition line of the chiral and confinementdeconfinement transitions. The arrows labeled $\mathrm{A}-\mathrm{C}$ indicate paths probed in Sec. IV.

transition at finite $\theta$ is not fully understood yet. First, there is no a priori reason that these two transitions coincide. LGT simulations, however, show that the two transitions take place at approximately the same temperature $[8,10,16]$. Second, the order of the transition depends on the quark mass and the number of flavors. For $N_{f}=2$ and $N_{c}=3$ (this case will be explored in this paper), Ref. [16] shows that for $m_{\pi} / m_{\rho} \simeq 0.9$ the chiral and confinementdeconfinement transition at $\theta=0.92(\pi / 3)$ is of the crossover type, implying that the transition at the RW end point is second-order. On the other hand, Refs. [17,23] have demonstrated that for light and heavy quark masses, the phase transition at $T_{E}$ is first-order. In this case, the first-order RW line continues along the dashed lines and terminates at a critical end point which is located at a value of the imaginary chemical potential range $0<\theta<\pi / N_{c}$.

In this paper, we do not attempt to obtain a fit of the PNJL model to lattice results at imaginary chemical potential. Rather, we focus on exploring qualitative features of the phase structure of the model and their origin.

\section{PNJL MODEL}

\section{A. Formulation}

Studies of the phase diagram of strongly interacting matter at imaginary chemical potential within effective models, requires a model with the same symmetry structure as QCD. The most important symmetries which must be accounted for are the extended $Z(3)$ and the chiral symmetries. In this article, we employ the Polyakovloop-extended Nambu-Jona-Lasinio (PNJL) model with $N_{f}=2$ and $N_{c}=3[27,28]$.

The Lagrangian of the two-flavor PNJL model with a four-quark interaction is given by

$$
\begin{aligned}
\mathcal{L}= & \bar{q}\left(i \gamma_{\mu} D^{\mu}-m_{0}\right) q+G_{s}\left[(\bar{q} q)^{2}+\left(\bar{q} i \gamma_{5} \vec{\tau} q\right)^{2}\right] \\
& -\mathcal{U}\left(\Phi[A], \Phi^{*}[A] ; T\right) .
\end{aligned}
$$

In the covariant derivative $D^{\mu}=\partial^{\mu}-i A^{\mu}$, only the temporal component $A_{0}$ of the gluon field $A_{\mu}=g A_{\mu}^{a} \lambda^{a} / 2$ is included. The gluon field is treated as a classical background field, whose dynamics is encoded in the effective potential $\mathcal{U}$. The gluon field is expressed in terms of the traced Polyakov loop and its conjugate

$$
\Phi=\frac{1}{3}\left\langle\operatorname{Tr}_{c} L\right\rangle, \quad \Phi^{*}=\frac{1}{3}\left\langle\operatorname{Tr}_{c} L^{\dagger}\right\rangle ;
$$

where the trace is taken over color space and

$$
L(\boldsymbol{x})=\mathcal{P} \exp \left[i \int_{0}^{\beta} d \tau A_{4}(\boldsymbol{x}, \tau)\right] .
$$

Here, $A_{4}=i A_{0}$ while $\mathcal{P}$ denotes the path ordering in Euclidean time $\tau$. In the Polyakov gauge, the matrix $L$ reduces to the diagonal form $L=\operatorname{diag}\left(e^{i \phi_{1}}, e^{i \phi_{2}}\right.$, $\left.e^{-i\left(\phi_{1}+\phi_{2}\right)}\right)$ [27]. Note that, in general, $\Phi^{*}$ is not the complex conjugate of $\Phi$. At real chemical potential, both $\Phi$ and $\Phi^{*}$ are real, and at $\mu \neq 0$ their values differ [41]. At imaginary chemical potential, $\Phi^{*}$ is the complex conjugate of $\Phi$ and they have nonzero imaginary parts [34]. Therefore, in the discussion below, we use the notation

$$
\begin{gathered}
\Phi=|\Phi| e^{i \phi}, \\
\Phi^{*}=|\Phi| e^{-i \phi}
\end{gathered}
$$

for imaginary $\mu$. The extended $Z$ (3) symmetry leads to the following properties of the Polyakov loop [34]:

$$
\left|\Phi\left(\theta+\frac{2 \pi k}{3}\right)\right|=|\Phi(\theta)|
$$

$$
|\Phi(-\theta)|=|\Phi(\theta)|
$$

$$
\begin{gathered}
\phi\left(\theta+\frac{2 \pi k}{3}\right)=\phi(\theta)-\frac{2 \pi k}{3}, \\
\phi(-\theta)=-\phi(\theta) .
\end{gathered}
$$

In the mean-field approximation, the thermodynamic potential can be written in terms of quark quasiparticles with a dynamical mass $M$, momentum $p=|\boldsymbol{p}|$, and energy $E_{p}=\sqrt{p^{2}+M^{2}}[27,34]$ 


$$
\begin{aligned}
& \Omega(T, V, \theta) \\
&=-4 V \int \frac{d^{3} p}{(2 \pi)^{3}}\left[3\left(E_{p}-E_{p}^{0}\right)+\frac{1}{\beta} \ln \left[1+3\left(\Phi+\Phi^{*} e^{-\beta E_{p}^{-}}\right)\right.\right. \\
&\left.\times e^{-\beta E_{p}^{-}}+e^{-3 \beta E_{p}^{-}}\right]+\frac{1}{\beta} \ln \left[1+3\left(\Phi^{*}+\Phi e^{-\beta E_{p}^{+}}\right) e^{-\beta E_{p}^{+}}\right. \\
&\left.\left.+e^{-3 \beta E_{p}^{+}}\right]\right]+\left(G_{s} \sigma^{2}+\mathcal{U}\right) V
\end{aligned}
$$

The term in the first line represents the ultraviolet divergent vacuum fluctuations. As usual, we introduce a threemomentum cutoff $\Lambda$ in the momentum integration to regularize the vacuum contribution. We subtract the vacuum term with the single-quark energy $E_{p}^{0}=\sqrt{p^{2}+m_{0}^{2}}$, so that the vacuum contribution vanishes when the chiral symmetry is restored, as in Ref. [41]. Moreover, we use a shorthand notation, where the imaginary chemical potential is subsumed in $E_{p}^{ \pm}=E_{p} \pm i \theta / \beta$. The dynamical mass $M$ is related to the current quark mass and the chiral condensate $\sigma=\langle\bar{q} q\rangle$ by $M=m_{0}-2 G_{s} \sigma$. The term $G_{s} \sigma^{2}$ in the last line is due to the meson potential in the Lagrangian (9).

So far, two types of the Polyakov-loop effective potential $\mathcal{U}$ have been widely used. The polynomial potential has as a general $Z(3)$ symmetric form $[28,42]$

$$
\frac{\mathcal{U}_{\text {poly }}}{T^{4}}=-\frac{b_{2}(T)}{2} \Phi^{*} \Phi-\frac{b_{3}}{6}\left[\Phi^{3}+\left(\Phi^{*}\right)^{3}\right]+\frac{b_{4}}{4}\left(\Phi^{*} \Phi\right)^{2}
$$

with

$$
b_{2}(T)=a_{0}+a_{1} \frac{T_{0}}{T}+a_{2}\left(\frac{T_{0}}{T}\right)^{2}+a_{3}\left(\frac{T_{0}}{T}\right)^{3} .
$$

The coefficients are determined by fitting the equation of state and the expectation value of the Polyakov loop to lattice data of pure gauge theory $[43,44]$ in Ref. [28]. In the other widely used variant, a logarithmic potential motivated by the strong coupling expansion is implemented $[27,45]$

$$
\begin{aligned}
\frac{U_{\log }}{T^{4}}= & -\frac{a(T)}{2} \Phi^{*} \Phi+b(T) \log \left\{1-6 \Phi^{*} \Phi+4\left[\Phi^{3}+\left(\Phi^{*}\right)^{3}\right]\right. \\
& \left.-3\left(\Phi^{*} \Phi\right)^{2}\right\}
\end{aligned}
$$

where

$$
a(T)=a_{0}+a_{1} \frac{T_{0}}{T}+a_{2}\left(\frac{T_{0}}{T}\right)^{2}, \quad b(T)=b_{3}\left(\frac{T_{0}}{T}\right)^{3} .
$$

The above parameterization for the temperature dependence was introduced in [45] and the constants are determined by fitting lattice data of pure SU(3) theory. In Ref. [27], a similar functional form in $\Phi$ but with parameterization of the temperature dependence was introduced. In this potential, however, one of the parameters is fixed to reproduce a simultaneous crossover transition for the chiral and deconfinement transitions rather than the first order
TABLE I. Parameters in the polynomial potential (19).

\begin{tabular}{lcccccc}
\hline \hline$T_{0}[\mathrm{MeV}]$ & $a_{0}$ & $a_{1}$ & $a_{2}$ & $a_{3}$ & $b_{3}$ & $b_{4}$ \\
\hline 270 & 6.75 & -1.95 & 2.625 & -7.44 & 0.75 & 7.5 \\
\hline \hline
\end{tabular}

TABLE II. Parameters in the logarithmic potential (21).

\begin{tabular}{lcccc}
\hline \hline$T_{0}[\mathrm{MeV}]$ & $a_{0}$ & $a_{1}$ & $a_{2}$ & $b_{3}$ \\
\hline 270 & 3.51 & -2.47 & 15.22 & -1.75 \\
\hline \hline
\end{tabular}

transition in the pure gauge theory. We refer to [46] for discussion. This difference makes it difficult to perform a systematic comparison of the effect of quarks near the deconfinement transition. If we refit the parameters to reproduce the pure $\mathrm{SU}(3)$ lattice data, we expect to have similar results to those from the logarithmic potential (21) since the target space and the transition temperature are the same. In this paper, we focus on the two potentials Eqs. (19) and (21) which equally reproduce the Polyakov loop and thermodynamics as well as the first-order confinement-deconfinement phase transition. We use the parameters determined in Refs. [28,45]. For convenience, they are summarized in Tables I and II.

The order parameters, chiral condensate $\sigma$ (or dynamical mass $M$ ), modulus of the Polyakov loop $|\Phi|$, and the phase of the Polyakov loop $\phi$ are determined numerically by solving the coupled equations of motion

$$
\frac{\partial \Omega(T, V, \theta ; M,|\Phi|, \phi)}{\partial X_{i}}=0, \quad X_{i}=M,|\Phi|, \phi .
$$

The phase diagram in the $T-\theta$ plane of the polynomial potential model (19) has been studied in Refs. [34,35]. In this model, a first-order Roberge-Weiss transition at $\theta=\pi / 3 \pm 2 \pi k / 3$, a second-order chiral transition in the chiral limit and a crossover one at finite quark masses as well as a crossover confinement-deconfinement transition were found. However, these features depend on the choice of the Polyakov-loop effective potential and further quark interaction terms are required to reproduce lattice results quantitatively $[24,35]$.

In this paper, we restrict ourselves to the simplest quarkquark interaction form, as shown in Eq. (9) and focus on the behavior of the order parameters in the $T-\theta$ plane for the polynomial and logarithmic Polyakov loop potentials.

\section{B. Some analytic insights}

Before proceeding to the full numerical computation, it is useful to explore the general properties of the thermodynamic potential (18) analytically in a few limiting cases. The momentum integration in Eq. (18) can be carried out analytically if we first expand the logarithmic terms in the integrand in powers of $e^{-\beta E_{p}} \ll 1$. We thus find, keeping terms up to order $\left(e^{-\beta E_{p}}\right)^{3}$, 


$$
\begin{aligned}
\Omega \simeq & \left(G_{s} \sigma^{2}+\mathcal{U}\right) V-\Omega_{0}-\frac{2 V}{\beta \pi^{2}}\left[3\left(\Phi e^{i \theta}+\Phi^{*} e^{-i \theta}\right) \int_{0}^{\Lambda} d p p^{2} e^{-\beta E_{p}}+\frac{3}{2}\left\{e^{2 i \theta}\left(2 \Phi^{*}-3 \Phi^{2}\right)+e^{-2 i \theta}\left(2 \Phi-3 \Phi^{* 2}\right)\right\}\right. \\
& \left.\times \int_{0}^{\Lambda} d p p^{2} e^{-2 \beta E_{p}}+\left\{2\left(1-9 \Phi \Phi^{*}\right) \cos 3 \theta+9 e^{3 i \theta} \Phi^{3}+9 e^{-3 i \theta} \Phi^{* 3}\right\} \int_{0}^{\Lambda} d p p^{2} e^{-3 \beta E_{p}}\right] .
\end{aligned}
$$

Here, $\Omega_{0}$ is the temperature independent vacuum term. While it is necessary to introduce a finite cutoff for this term, due to the nonrenormalizability of the PNJL model, taking $\Lambda \rightarrow \infty$ in the thermal part does not affect the qualitative features discussed below. Thus the momentum integration of the thermal part can be carried out explicitly, resulting in modified Bessel functions $K_{n}$.

We first consider the low temperature limit $\Phi=$ $\Phi^{*}=0$ in order to explore the effect of the Polyakov loop in the confined phase. In this case, the Polyakov loop effective potential, $\mathcal{U}$, vanishes. Furthermore, the gap equation for the dynamical mass $M$, obtained from Eq. (24), $\partial \Omega / \partial M=0$, reduces to

$$
\begin{aligned}
M \simeq & m_{0}+\frac{6 G_{s}}{\pi^{2}}\left[M^{3} f(\Lambda / M)-m_{0}^{3} f\left(\Lambda / m_{0}\right)\right] \\
& -\frac{8 G_{s} M^{2} T \cos 3 \theta}{\pi^{2}} K_{1}(3 M / T)
\end{aligned}
$$

where

$$
f(x)=x \sqrt{x^{2}+1}-\ln \left(x+\sqrt{x^{2}+1}\right) .
$$

Note that, for $3 M / T \gg 1$,

$$
K_{1}(3 M / T) \sim \sqrt{\frac{\pi T}{6 M}} e^{-3 M / T} .
$$

The gap equation, Eq. (25), implies that the $\theta$ dependence of the dynamical mass is completely determined by $\cos 3 \theta$ term. Consequently, $M$ is a periodic function of $\theta$ with the period $2 \pi / 3$, as expected. For small temperatures, quark degrees of freedom are strongly suppressed, $\sim e^{-3 M / T}$, since, for a vanishing Polyakov loop, only three-quark clusters survive. The chiral phase transition takes place when the thermal contribution in the gap equation is of the same order as the vacuum term. If the condition $\Phi=\Phi^{*}=0$ is strictly enforced, the chiral transition is shifted to very high temperatures. Because the thermal excitation of quarks in this limit is possible only in threequark clusters, the resulting thermodynamics is qualitatively similar to that of the nucleonic NJL model, which also yields a very high chiral transition temperature [47].

In the (naive) high-temperature limit, $\Phi=\Phi^{*} \rightarrow 1$, the gap equation reduces to that of the ordinary NJL model,

$$
\begin{aligned}
M= & m_{0}+\frac{6 G_{s}}{\pi^{2}}\left[M^{3} f(\Lambda / M)-m_{0}^{3} f\left(\Lambda / m_{0}\right)\right] \\
& -\frac{24 G_{s} M^{2} T \cos \theta}{\pi^{2}} K_{1}(M / T) .
\end{aligned}
$$

Now, the $\theta$ dependence of the dynamical mass is determined by $\cos \theta$ and the thermal factor is proportional to $K_{1}(M / T)$, appropriate for the thermal excitation of single quarks. In this case, the dynamical mass has lost the original periodicity of the partition function (8), which is respected in the low-temperature limit (25).

At $\theta=\pi / 2$, the thermal contribution in (28) vanishes and consequently the dynamical mass equals its vacuum value, irrespective of temperature. Hence, in this approximation the phase boundary is shifted to higher temperatures as the imaginary chemical potential is increased, and eventually approaches $T=\infty$ in the limit $\theta \rightarrow \pi / 2$. In general, the thermal contribution is of the form $-\sum_{n} L_{n} \cos n \theta K_{1}(n M / T)$ with $L_{n}>0$. Because of the higher order terms, the transition temperature remains finite, but the positive curvature of the phase boundary persists. ${ }^{1}$ On the other hand, for real chemical potentials the leading thermal contribution is proportional to $T \cosh \beta \mu$. Since $\cosh x$ is an increasing function of $x$, this implies that the chiral transition temperature decreases as the real chemical potential grows.

In the high-temperature limit, as implemented above, the original periodicity of the partition function is lost because the phase of the Polyakov loop is neglected. In fact, at imaginary chemical potential, the high temperature limit of the PNJL model is in general not the NJL model. The Roberge-Weiss transition, characterized by discontinuous jumps of the phase $\phi$, preserves the periodicity $2 \pi / 3$ in the deconfinement phase.

\section{BEHAVIOR OF THE ORDER PARAMETERS AT IMAGINARY CHEMICAL POTENTIAL}

We now discuss the characteristics of the order parameters obtained by solving the full gap equation Eq. (23). Besides the Polyakov loop potentials given in the previous section, the model has three parameters in the fermion sector. We use

$$
\begin{aligned}
G_{s} & =5.498 \mathrm{GeV}^{-2}, \\
\Lambda & =0.6315 \mathrm{GeV},
\end{aligned}
$$

which reproduce the vacuum pion mass and pion decay constant at zero temperature and density, when the current quark mass is fixed to the value $m_{0}=5.5 \mathrm{MeV}$ [31].

In what follows, we compare the results obtained for a finite pion mass with those corresponding to the chiral limit, $m_{0}=0$. In the latter case, the model belongs to the universality class of the three-dimensional $O(4)$ spin model

\footnotetext{
${ }^{1}$ Owing to the reflection symmetry and periodicity, it is sufficient to consider the interval $0 \leq \theta \leq \pi / 3$.
} 
MORITA et al.

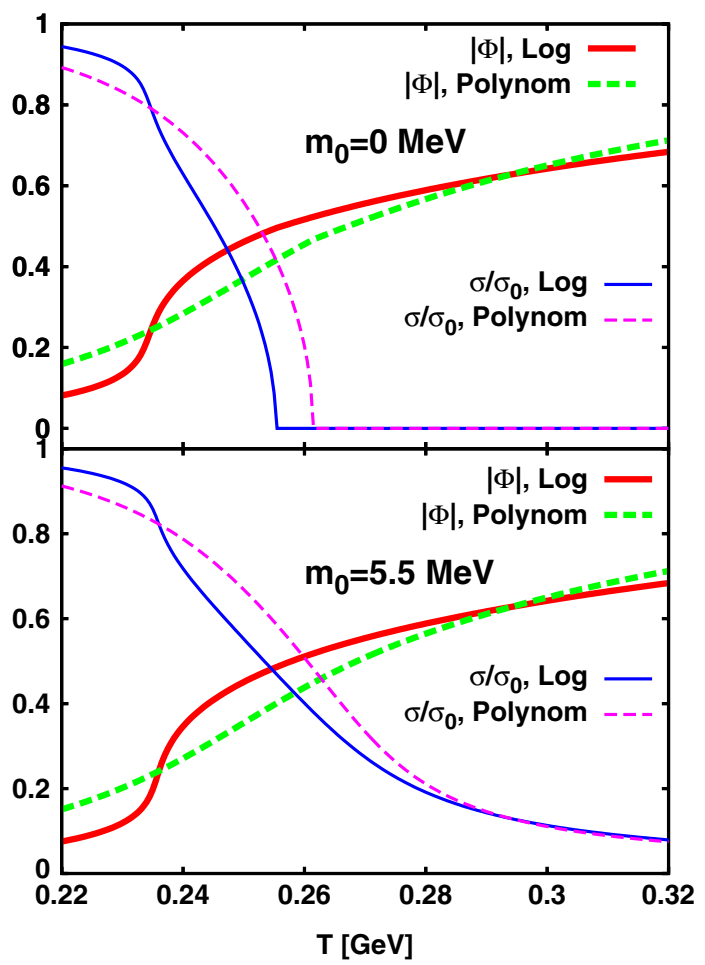

FIG. 2 (color online). Temperature dependence of the chiral condensate normalized by the value at $T=0$ and the Polyakov loop at vanishing chemical potential. The upper panel shows the result in the chiral limit while the lower stands for the physical quark mass.

and exhibits a second-order phase transition at finite temperature and small values of the real chemical potential.

With the parameter set given above, we find the chiral condensate and the Polyakov loop shown in Fig. 2. The dependence of the order parameters on the temperature shows that in the chiral limit, the chiral transition is indeed second order, while the confinement-deconfinement transition is of the crossover type. For finite quark mass, $m_{0}=5.5 \mathrm{MeV}$, shown in the lower panel, the chiral order parameter and the Polyakov loop both exhibit smooth crossover transitions. Thus, the explicit symmetry breaking induces a qualitative change of the chiral condensate, while for the Polyakov loop this dependence is negligible. Furthermore, a comparison of the two parametrizations of the Polyakov-loop effective potential shows that the transition is smoother for the polynomial potential than for the logarithmic one.

\section{A. Behavior across $\theta=\pi / 3$}

We now consider the order parameter as a function of $\theta$ close to $\theta=\pi / 3$. In Fig. 3, we show the phase of the Polyakov loop $\phi$ as a function of $\theta$ in the chiral limit. We do not show results for nonzero quark mass, since the results are indistinguishable from those shown in Fig. 3.

At low temperatures (arrow A in Fig. 1), the phase of the Polyakov loop changes smoothly from 0 at $\theta=0$ to $-\pi / 3$
PHYSICAL REVIEW D 84, 076009 (2011)

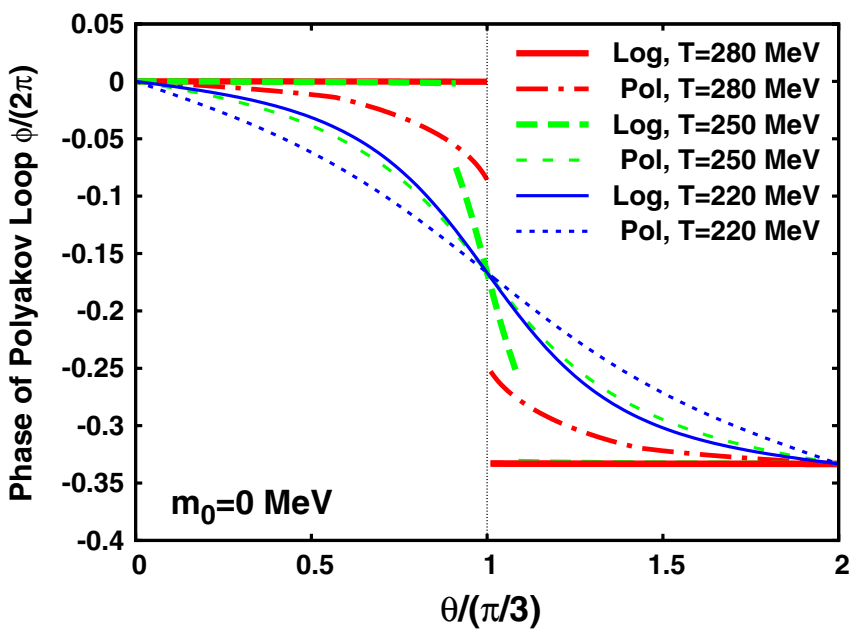

FIG. 3 (color online). Phase of Polyakov-loop as a function of $\theta$ for various temperatures in the chiral limit. As in Fig. 2, each line stands for the results of the logarithmic Polyakov-loop potential and the polynomial one at the different temperatures, respectively.

at $\theta=\pi / 3$. Subsequently, the phase continues to decrease and finally approaches to $-2 \pi / 3$ at $\theta=2 \pi / 3$, as required by the symmetry, Eq. (16). This behavior is independent of the choice of $\mathcal{U}$. At temperatures beyond $T_{E}$, the transition is first order (arrow C in Fig. 1). For instance, at $T=280 \mathrm{MeV}$ the phase jumps from $\phi=0$ to $-2 \pi / 3$ at $\theta=\pi / 3$. This is the Roberge-Weiss transition [5], where the phase of the Polyakov loop jumps from one $Z(3)$ sector to another. The RW transition is common to both parametrizations of $\mathcal{U}$. This is natural, since the RW transition is a consequence of the $Z$ (3) symmetry of the pure gauge theory, which is incorporated in both potentials. The detailed behavior around $T_{E}$ is, however, different between the two potentials. Thus, at $T=250 \mathrm{MeV}$ for the logarithmic potential, which is below the end point of the RW transition $\left(T_{E} \simeq 255 \mathrm{MeV}\right)$, the phase is discontinuous at $\theta \neq \pi / 3$. This implies that the phase boundary, which is crossed by arrow B in Fig. 1, is first-order at this temperature. By contrast, in the polynomial case the phase is a smooth function of $\theta$ at any temperature below the RW end point $\left(T_{E} \simeq 275 \mathrm{MeV}\right)$. As we discuss below, this reflects the different order of the RW end point for the two potentials.

We note that the logarithmic potential is defined in a limited domain, characterized by positivity of the argument of the logarithm, while for the polynomial potential there is no such restriction. In fact, for high temperatures (e.g. $T=280 \mathrm{MeV}$ ) the Polyakov loop, plotted for the polynomial potential as a function of $\theta$ in the complex $\Phi$ plane, leaves the so-called target space, defined by requiring that the logarithmic potential is real [48].

In Fig. 4, we show the modulus of the Polyakov-loop $|\Phi|$ as a function of $\theta$. At high temperatures, the RW transition is manifested by a cusp at $\theta=\pi / 3$, while at lower temperatures $|\Phi|$ varies smoothly for both parametrizations 


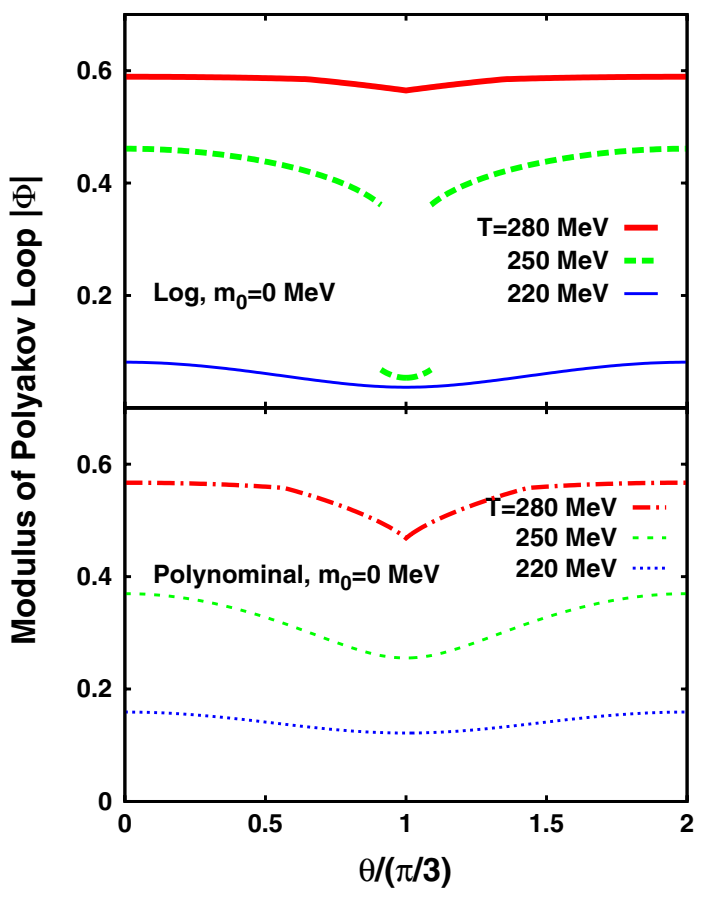

FIG. 4 (color online). Modulus of Polyakov loop as a function of $\theta$ in the chiral limit. Upper panel: Logarithmic potential. Lower panel: Polynomial potential. The temperatures are the same as in Fig. 3.

of the Polyakov-loop potential. However, at intermediate temperatures, the two potentials yield qualitatively different results, as illustrated by the discontinuities in $|\Phi|$ obtained for the logarithmic potential near $\theta=\pi / 3$ at $T=250 \mathrm{MeV}$. For the polynomial potential we find a continuous confinement-deconfinement transition at imaginary chemical potential, while for the logarithmic potential the transition is first-order at intermediate temperatures. We return to this point in the following subsection. Here we note only that the first-order transition is reflected also in a sudden change of the phase at $T=250 \mathrm{MeV}$, as shown in Fig. 3.

Within the PNJL model, the transition from one $Z(3)$ sector to another can be understood in the following way. In the high-temperature limit, the dominant contribution to the thermodynamic potential is given by the single-quark excitation term in Eq. (24) (the first term in the square bracket), which yields a contribution to $\Omega \sim-\cos (\theta+\phi)$. The only additional $\phi$-dependent term in $\Omega$ is the Polyakov-loop potential $\mathcal{U}$. At high temperature, $\mathcal{U}$ has the three local minima at $\phi=0$ and $\pm 2 \pi / 3$. For each value of $\theta$, the physical vacuum is obtained by finding the absolute minimum of the two terms. As illustrated in Fig. 5, the physical vacuum changes from one minimum to the next as $\theta$ crosses $\pi / 3+2 \pi k / 3$. While both potentials have the periodicity $\Delta \theta=2 \pi$, when $\phi$ is artificially fixed in one $Z(3)$ sector, the complete thermodynamic potential acquires the periodicity $\Delta \theta=2 \pi / 3$ owing to the $\phi$ dependence of $\mathcal{U}$.

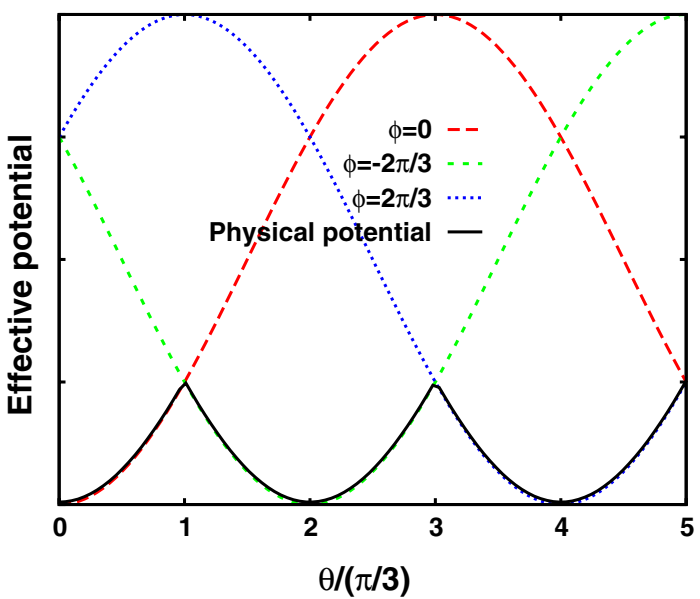

FIG. 5 (color online). Schematic effective potential for the RW transition.

Similarly, the chiral condensate $\sigma$ is also continuous near $\theta=\pi / 3$ at temperatures lower than $T_{E}$, irrespective of the quark mass and the potential, as shown in Fig. 6. At temperatures higher than $T_{E}$, it develops a cusp for both parametrizations, a manifestation of the RW transition. Furthermore, at temperatures above $T_{\chi}(\theta=\pi / 3)$, $\sigma$ vanishes for any value of $\theta$ in the chiral limit, as shown for $T=340 \mathrm{MeV}$. For a finite quark mass, the cusp persists to temperatures much higher than $T_{\chi}$ but finally

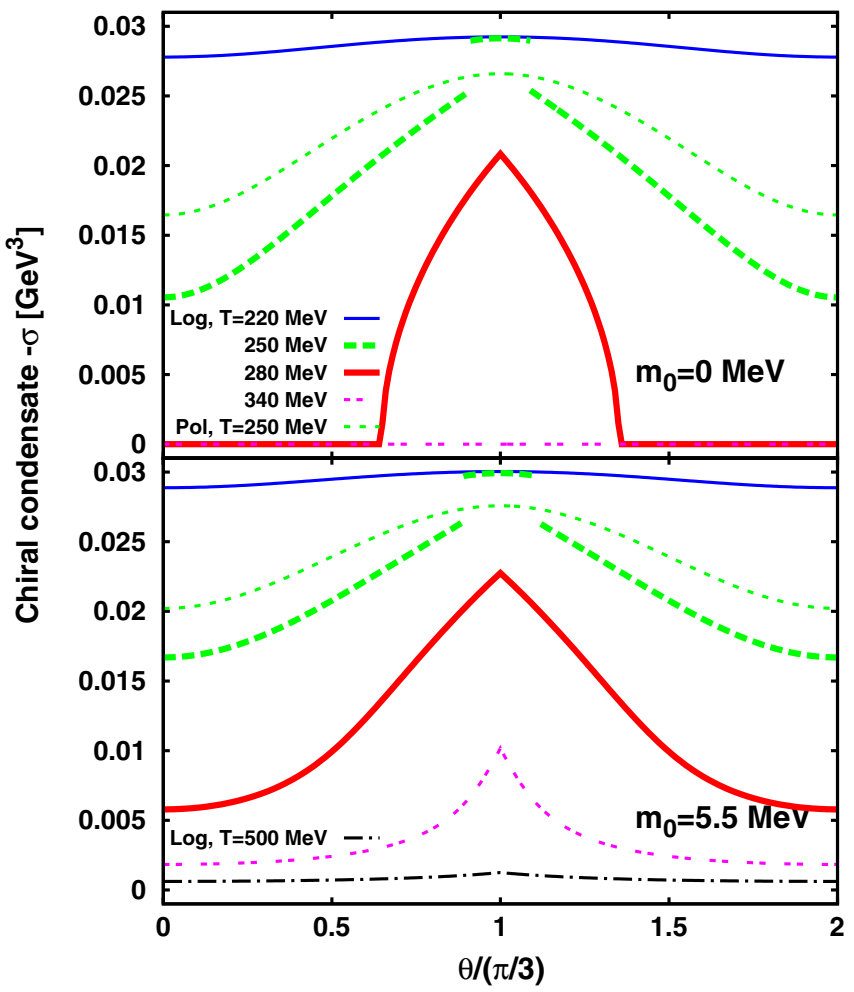

FIG. 6 (color online). Chiral condensate at various temperatures as a function of $\theta$ in the chiral limit. The temperatures associated with the lines are the same as Fig. 3. 
disappears, as shown in Fig. 6 for $m_{0}=5.5 \mathrm{MeV}$ and $T=500 \mathrm{MeV}$.

In the chiral limit, for temperatures in the interval between $T_{\chi}(\theta=0)$ and $T_{\chi}(\theta=\pi / 3)$, there is a second-order chiral transition at nonzero $\theta$, as shown in Fig. 6, (see also arrow B in Fig. 1). For finite quark masses, this transition is of the crossover type, as illustrated in the lower panel of Fig. 6 for $m_{0}=5.5 \mathrm{MeV}$. Also, the temperature dependence of $\sigma$ is clearly different for the two potentials. In particular, there is a discontinuity in $\sigma$ near $\theta=\pi / 3$ at $T=250 \mathrm{MeV}$ for the logarithmic potential. The values of temperature and imaginary chemical potential corresponding to the discontinuity are identical to the ones obtained for the Polyakov loop.

\section{B. First-order phase transition at $T<T_{E}$}

In this section, we focus on the first-order transition found in a limited temperature range below the RobergeWeiss transition for the logarithmic potential. In Fig. 7, we illustrate this result at $T=250 \mathrm{MeV}$. In each panel, two lines are shown: one is the solution of the gap equations approaching the transition from small $\theta$, while the other is obtained by approaching from the opposite side.

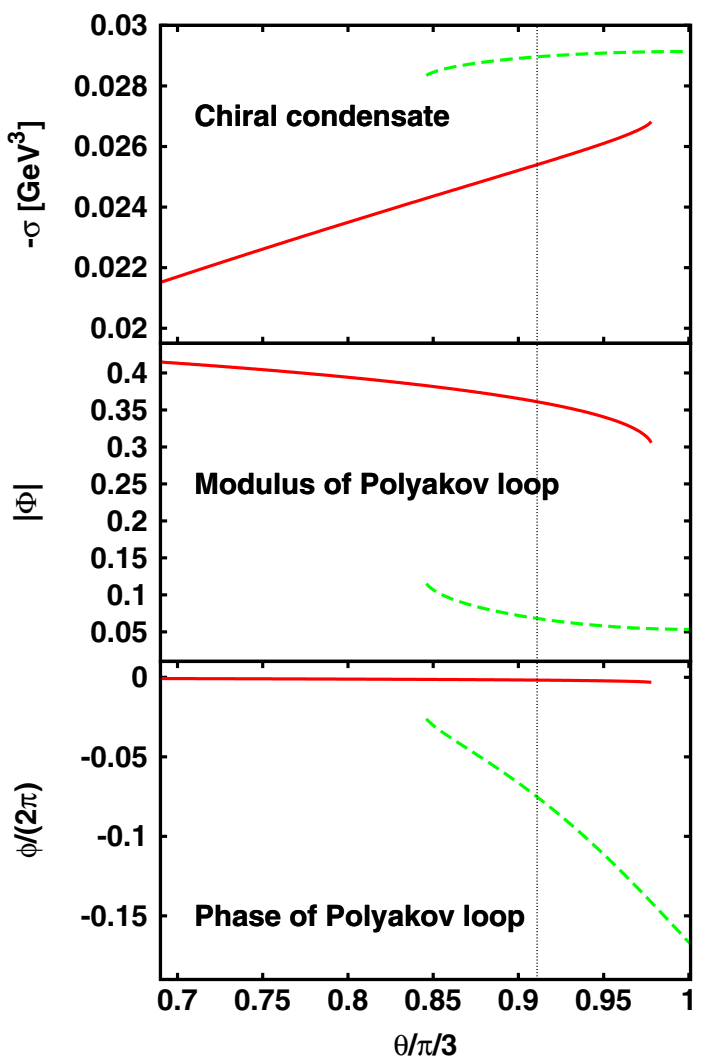

FIG. 7 (color online). Behavior of the order parameters around a first-order phase transition point in the chiral limit. We depict $T=250 \mathrm{MeV}$. From top to bottom, each panel shows the chiral condensate, modulus of the Polyakov loop, and the phase of the Polyakov loop. The vertical line (thin dashed) shows the critical $\theta$, determined by a Maxwell construction.
The existence of two solutions in a certain range of $\theta$ shows that there are two local minima in the thermodynamic potential. The first-order phase transition takes place at the value of $\theta$, where the thermodynamic potential in the two local minima is degenerate. At $T=250 \mathrm{MeV}$, this happens at $\theta=0.911(\pi / 3)$. The lines terminate where the corresponding minimum disappears. Thus, the system exhibits hysteresis, a characteristic of a first-order phase transition.

Although this transition is related to the confinementdeconfinement transition, the discontinuity is reflected also in the chiral condensate $\sigma$, owing to the coupling between the Polyakov loop and the chiral order parameter.

\section{The RW end point}

The existence of the first-order transition, discussed in the previous section, is closely related to the characteristics of the RW end point. In Fig. 8, we show the temperature dependence of the Polyakov loop along the line $\theta=\pi / 3$ (cf. Fig. 1) for the two potentials in the chiral limit. The nature of the RW end point, as characterized e.g. by the phase of the Polyakov loop, differs between the two potentials. While the polynomial potential yields a continuous transition, the logarithmic one exhibits a discontinuity in the phase and magnitude of the Polyakov loop.

Above the RW end point $\left(T=T_{E}\right)$, the phase of the Polyakov loop can take two values on the $\theta=\pi / 3$ line, corresponding to different $Z$ (3) sectors (cf. Fig. 5). Thus, in the case of the polynomial potential, the phase bifurcates

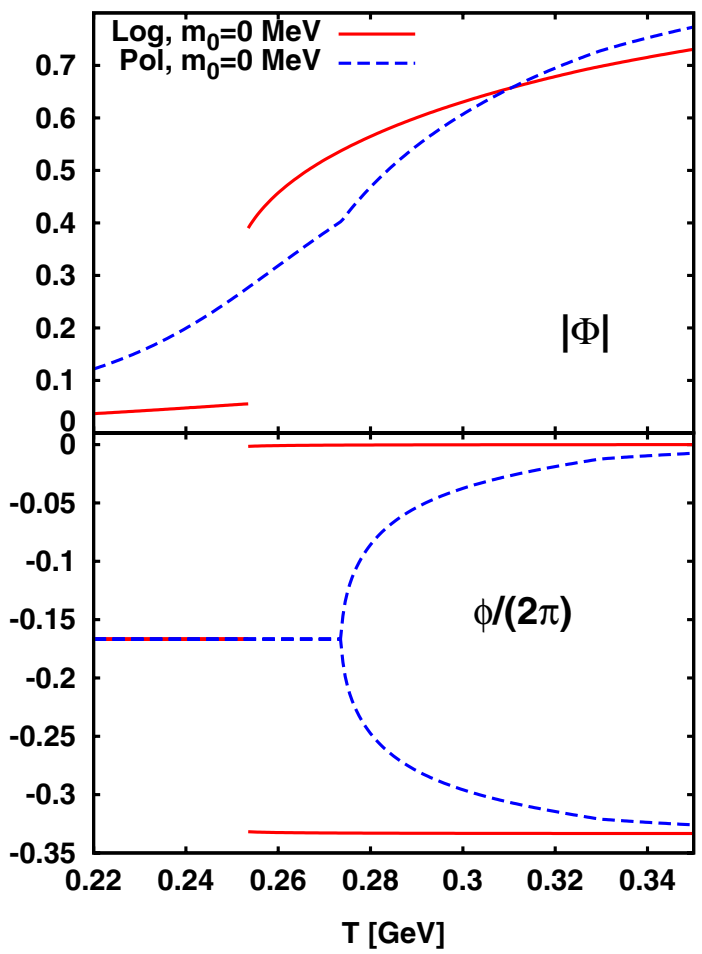

FIG. 8 (color online). Behavior of $|\Phi|$, and $\phi$ as functions of $T$ at $\theta=\pi / 3$. 
smoothly at the RW end point, while for the logarithmic potential, the phase changes discontinuously at this point. In the former case, the RW end point is a second-order point, while in the latter it is a triple point. We note, however, that a different parameterization for the logarithmic potential [27] also yields a second-order RW end point [22].

Consequently, the characterization of the RW end point depends on the parametrization of the Polyakov-loop potential, but, within the framework considered here, it is independent of the value for the quark mass for the logarithmic potential. On the other hand, in LGT calculations it is found that the nature of the RW end point depends crucially on the quark mass; for both two- and three-flavor QCD, it is a second-order end point at intermediate quark masses and a triple point for large and small masses [17,25]. In Ref. [24], it is argued that in order to reproduce the quark mass dependence of the RW transition, a $\Phi$ dependent fermion coupling, motivated by functional renormalization group analyses $[49,50]$, is required. This indicates that in QCD the interplay between chiral symmetry breaking and confinement is more complicated than in the present model.

Finally, in Fig. 9 we summarize the results on the phase diagram in the $T-\theta$ plane for the two Polyakov-loop potentials. The (pseudo-)critical temperature for the
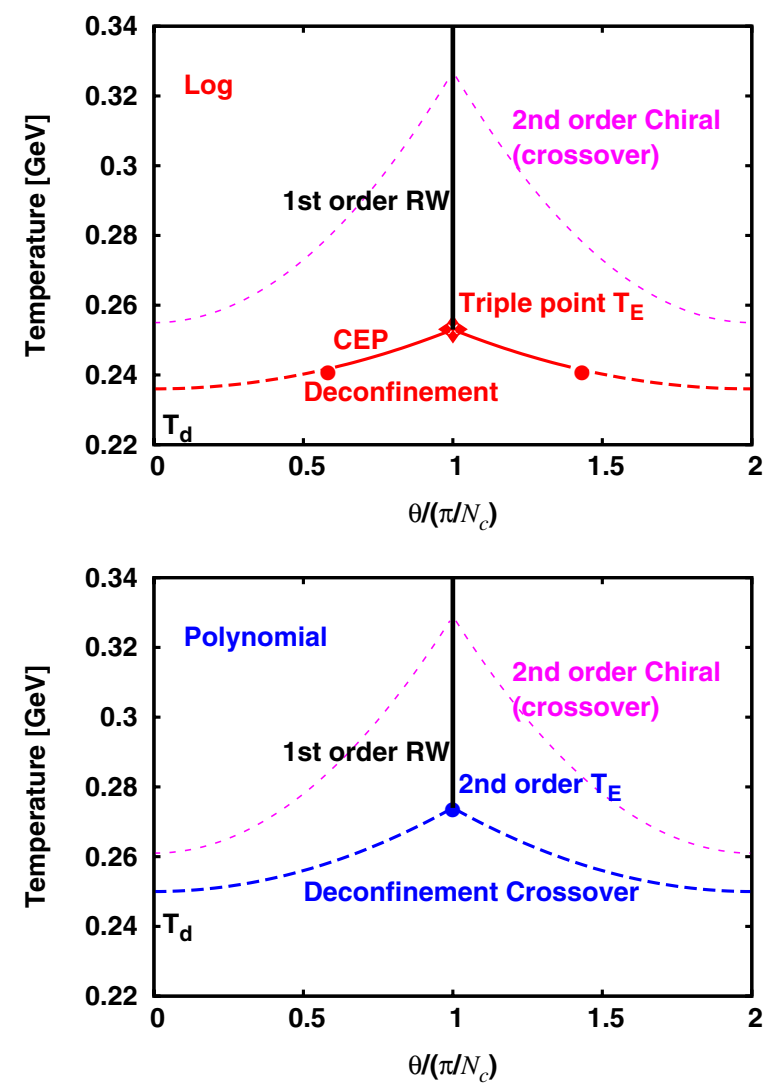

FIG. 9 (color online). Phase diagram for the logarithmic potential (top) and the polynomial potential (bottom). deconfinement transition corresponds to a maximum of the temperature derivative of the modulus of the Polyakov-loop $d|\Phi| / d T$. The critical end point (CEP), obtained for the logarithmic potential at $0<\theta<\pi / 3$, is a consequence of the triple point at $T=T_{E}$ and $\theta=\pi / 3$.

In comparison of the two potentials, we have seen that the phase transitions at imaginary chemical potential are shifted to higher temperatures compared to those at real chemical potential. This implies that dynamical quark mass becomes heavier at fixed temperature (see Fig. 6), thus the Polyakov-loop potential $\mathcal{U}$, which is independent of $\theta$, makes a dominant contribution to the thermodynamic potential. Furthermore, at imaginary chemical potential, the target space of the Polyakov loop is probed through the change of the phase $\phi$. Therefore, a comparison of the resulting phase diagram at the imaginary chemical potential region with that obtained in LGT calculations, yields important constraints on the effective Polyakov-loop potential.

\section{Critical end point of confinement-deconfinement transition}

In Ref. [51], it was found that as the pion mass in the quark-meson model is reduced from its physical value, an additional critical end point appears on the phase boundary at small (real) values of the chemical potential. Since the coupling to the Polyakov loop is not accounted for in [51], the additional CEP is associated with the chiral phase transition. ${ }^{2}$ In this section, we explore the dependence of the confinement-deconfinement CEP, which appears at imaginary $\mu$ for the logarithmic potential, on the model parameters. We find that the location of this CEP depends on the four fermion coupling constant $G_{s}$.

In Fig. 10, we show the phase diagram of the model in the chiral limit for different values of $G_{s}$. We include both real and imaginary values of $\mu$ by showing the phase boundaries in $T-\mu^{2}$ plane. The upper panels show cases where $G_{s}$ is smaller, while the lower ones show cases where it is larger than or equal to the reference value (29). Lines appearing for $\mu^{2}<-(\pi T / 3)^{2}$ (the boundary is indicated by the dotted line) are images of those in the region $-(\pi T / 3)^{2} \leq \mu^{2} \leq 0$; the mapping is defined by the periodicity of the partition function.

We identify the (pseudo)critical temperature by finding the maximum of the derivative of the corresponding order parameter with respect to temperature. For real values of the chemical potential, the Polyakov loop and its conjugate are real but take on different values [41]. Here, we use $d \Phi / d T$ for the definition of the deconfinement transition. A different definition, based e.g. on the Polyakov-loop

\footnotetext{
${ }^{2}$ The fact that the model calculation of Ref. [51] yields a firstorder chiral transition at both small and large values of $\mu$ in the chiral limit, is presumably due to the neglect of the fermion vacuum loop [52].
} 


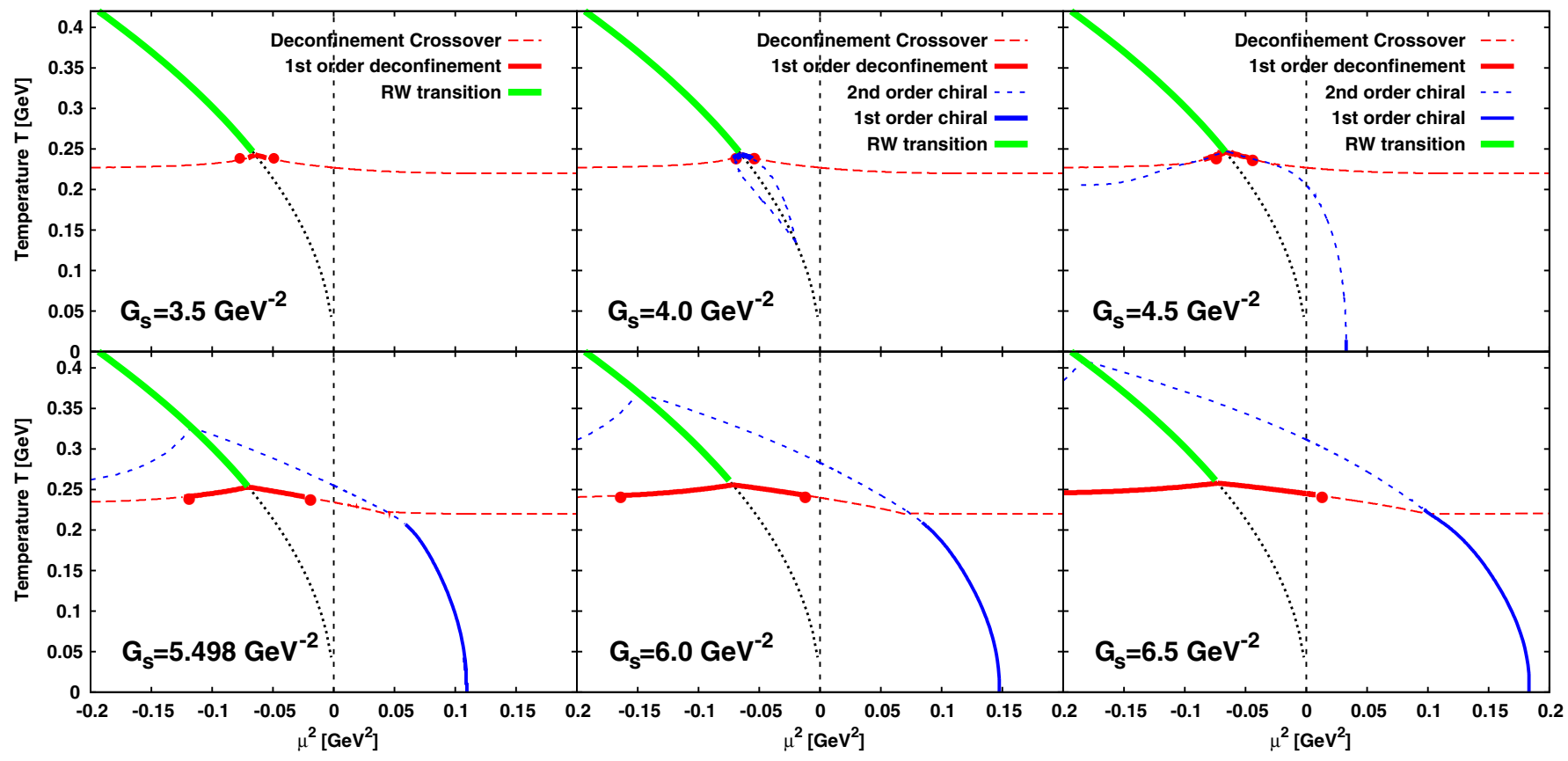

FIG. 10 (color online). Phase diagrams on $T-\mu^{2}$ plane for various $G_{s}$ in the chiral limit. The solid lines stand for the first-order phase transition lines. Dashed lines denote the second order and crossover transitions. For convenience, $\mu^{2}=0$ and $\mu_{I} / T=\pi / 3$ are indicated by the thin and thick dotted lines, respectively. The filled circles show the location of the CEP for the confinementdeconfinement transition.

susceptibility [41], would lead to a slightly different value of the pseudocritical temperature of the crossover transition.

For the first-order transition at large $\mu^{2}$, one finds double peaks in $d \Phi / d T$ (cf. Fig. 10). Here, we identify the position of the transition with the maximum which smoothly extrapolates to the deconfinement transition at vanishing chemical potential and to the chiral transition at $T=0$, where the peak structure is simple. We note that any ambiguity in the location of the phase boundary does not affect the discussion below.

Qualitatively the features of the phase diagram can be classified as follows: The NJL sector has a critical coupling $G_{s}^{\mathrm{cr}}=\pi^{2} /\left(2 N_{c} \Lambda^{2}\right)$ for the gap equation to have a nontrivial solution [31]. This implies that, with the present three-momentum cutoff, there is no spontaneous breakdown of chiral symmetry for $G_{s}<4.125 \mathrm{GeV}^{-2}$. Therefore, in the upper-left panel $\left(G_{s}=3.5 \mathrm{GeV}^{-2}\right)$, the system is everywhere in the chirally symmetric phase. In this case, the RW end point is still a triple point, and the CEP of the deconfinement transition is close by, at $\theta=0.95 \pi / 3$.

For $G_{s}>G_{s}^{\mathrm{cr}}$, there is a chiral transition at vanishing chemical potential. As seen in the upper-center panel, there is a precursor at imaginary chemical potential for $G_{s}$ slightly smaller than $G_{s}^{\mathrm{cr}}$. The chiral symmetry is spontaneously broken in a small region at intermediate temperatures adjoining the $\mu^{2}=-(\pi T / 3)^{2}$ line. This behavior can be understood along the lines presented in Sec. III B. Although the Boltzmann approximation, Eq. (24), might not be a good approximation since the system is in the chirally symmetric phase even at low temperatures, the Polyakov loop is small so that the thermal contribution is dominated by the $\cos 3 \theta$ term as in Eq. (25). Since this term is positive in the $\pi / 6<\theta<\pi / 2$, it adds to the vacuum term and a nontrivial solution appears at finite temperature where the system enters the broken phase.

As the temperature is increased further, however, the Polyakov loop is nonzero and the one- and two- quark excitations contribute to the gap equation, driving the system back into the symmetric phase. Consequently, near the RW end point, the chiral and deconfinement transitions occur simultaneously and the chiral transition is also of first order. Note that the lower end point of the chiral transition follows the $\mu^{2}=-(\pi T / 3)^{2}$ line and arrives at the origin when $G_{s}=G_{s}^{\mathrm{cr}}$. For $G_{s}$ beyond this value, the chiral transition line enters the $\mu^{2}>0$ half-plane.

As $G_{s}$ is increased beyond $G_{s}=G_{s}^{\text {cr }}$, the location of the deconfinement CEP moves to larger $\mu^{2}$ and finally reaches real values of the chemical potential at $G_{s} \simeq 6.5 \mathrm{GeV}^{-2}$. At the same time, the chiral transition line moves to larger $T$ and $\mu^{2}$. The behavior of the CEP can be directly related to changes of the chiral transition with increasing $G_{s}$. Increasing $G_{s}$ leads to a larger dynamical mass $M=m_{0}$ $2 G_{s} \sigma$ in the chirally broken phase. Moreover, $|\sigma|$ increases with $G_{s}$ since a stronger scalar coupling leads to larger quark condensate. This raises both the dynamical mass and the chiral transition temperature. On the other hand, a modified $G_{s}$ does not alter the Polyakov-loop sector of 


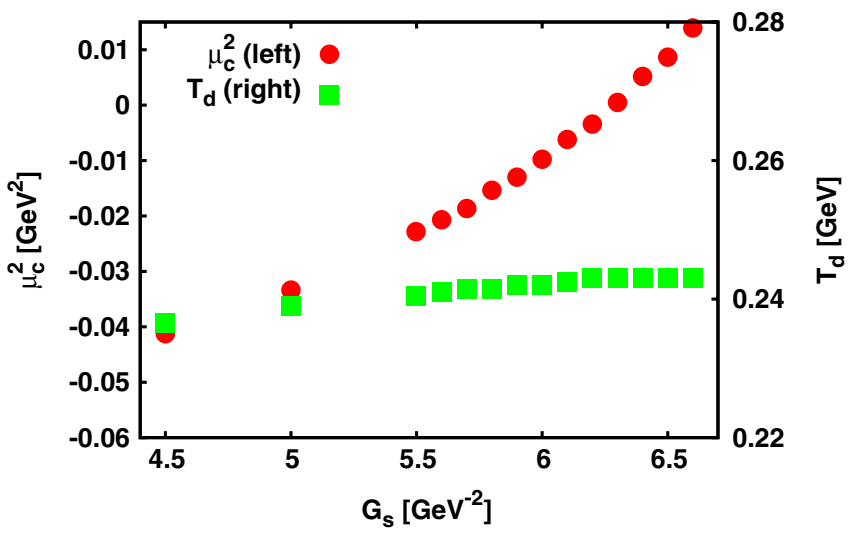

FIG. 11 (color online). Location of the CEP as a function of $G_{s}$. The left vertical axis shows the chemical potential while the right one denotes the temperature.

the model. Consequently, near the deconfinement transition, the dynamical mass of the quarks increase with $G_{s}$ and the system approaches a pure gauge theory owing to the thermal suppression of quark degrees of freedom. Thus, as shown in Fig. 11, the first-order phase transition of the pure gauge theory is recovered at $G_{s}=6.3 \mathrm{GeV}^{-2}$, only $15 \%$ above the reference value (29).

One may wonder why this mechanism is not effective for the polynomial potential, since it also exhibits a first-order phase transition in the absence of fermions. In fact, we find that the polynomial potential does show the same behavior, but at much larger values of the scalar coupling. The RW end point, which is second-order at $G_{s}=5.498 \mathrm{GeV}^{-2}$, is first-order starting at $G_{s}=12.4 \mathrm{GeV}^{-2}$, and the deconfinement CEP reaches $\mu^{2}=0$ at $G_{s}=25 \mathrm{GeV}^{-2}, 4.5$ times larger than the reference value.

The origin of this quantitative difference between the polynomial and logarithmic potentials is due to the much weaker first-order phase transition (smaller discontinuity in $\Phi$ ) exhibited by the polynomial potential in the heavy quark limit. At $G_{s}=25 \mathrm{GeV}^{-2}$, we find a dynamical quark mass of about $2.5 \mathrm{GeV}$. Thus, for a quark mass less than $2.5 \mathrm{GeV}$ the first-order transition is smoothened to a crossover transition. By contrast, for the logarithmic potential this happens at a much smaller dynamical quark mass of $0.4 \mathrm{GeV}$, owing to the much stronger underlying first-order transition.

We note that a first-order confinement-deconfinement transition emerges at real chemical potential also in the large $N_{c}$ limit of the PNJL model [53], as explored in the context of the recently proposed quarkyonic phase [54]. Indeed, the effect of strengthening $G_{s}$ is similar to that of increasing $N_{c}$ since $G_{s}$ and $N_{c}$ appear in the factor $G_{s} N_{c}$ in the gap equation for the dynamical mass (see Eq. (16) in Ref. [53]). While we suppress the quark contribution to the thermodynamics by increasing the dynamical mass by means of $G_{s}$, a large value of $N_{c}$ is accompanied by a $1 / N_{c}$ suppression of the quark contribution. Both procedures yield a gluon dominated system and thus give a first-order confinementdeconfinement transition.

Note, however, that the two procedures differ in detail. Increasing $G_{s}$ at fixed $N_{c}$ preserves the Polyakov-loop potential but modifies the quark mass, while in the large $N_{c}$ limit at fixed $G_{s} N_{c}$ the quark mass remains unchanged but the Polyakov-loop potential is modified. This means that when we increase $G_{s}$ at fixed $N_{c}$ we change the characteristic scale of the chiral symmetry breaking. Although this does not correspond to the physical situation, since QCD has a unique scale, $\Lambda_{\mathrm{QCD}}$, our result could be useful for exploring the interplay between the chiral phase transition and deconfinement.

\section{DUAL PARAMETER FOR THE CONFINEMENT-DECONFINEMENT TRANSITION}

In this section, we consider dual parameters which capture the characteristic feature of the confinementdeconfinement transition discussed above. Recently, a dual parameter has been introduced by considering a generalized boundary condition for fermions

$$
q(\boldsymbol{x}, \boldsymbol{\beta})=e^{i \varphi} q(\boldsymbol{x}, 0) .
$$

Here, $\varphi$ is the so-called twisted angle. The dual quark condensate $\Sigma^{(n)}$ is defined as the $n$-th Fourier coefficient of the chiral condensate as a function of the twisted angle [40]

$$
\Sigma^{(n)}(T)=-\int_{0}^{2 \pi} \frac{d \varphi}{2 \pi} e^{-i n \varphi} \sigma(T, \varphi) .
$$

The chiral condensate $\sigma(T, \varphi)$ is defined in terms of $\varphi$ by [40]

$$
\sigma(\varphi)=-\frac{1}{V}\left\langle\operatorname{Tr}\left[\left(m_{0}+D_{\varphi}\right)^{-1}\right]\right\rangle_{G}
$$

In Ref. [40], this quantity was introduced based on the lattice regularization. The $\varphi$ dependence of $\operatorname{Tr}\left[\left(m_{0}+D_{\varphi}\right)^{-1}\right]$ can be written down explicitly by using the link variable. It reduces to the ordinary chiral order parameter in the limit of $m \rightarrow 0$ and $V \rightarrow \infty$. An implementation in the continuum theory has been done in the framework of Dyson-Schwinger equation [55]. The most interesting quantity is that of $n=1$, which is called dressed Polyakov loop. Because of the relation to the ordinary Polyakov loop, it can be regarded as an order parameter of the confinement-deconfinement transition.

From the fermionic boundary conditions (31), one immediately finds that this is equivalent to introducing the imaginary chemical potential [Eq. (5)]. The only difference is that $\varphi= \pm \pi$ corresponds to the usual antiperiodic boundary condition in the twisted angle while $\theta=0$ does so in the imaginary chemical potential. In this case, the relation between $\varphi$ and $\theta$ is given by just a shift of $\pm \pi$,

$$
\varphi=\theta \pm \pi \quad\left(\bmod 2 \pi / N_{c}\right)
$$


Furthermore, one notes that the definition of dual condensate (32) is formally similar to the projection on the canonical partition function (1).

However, the LGT calculations demonstrate that $\sigma(\varphi)$ exhibits quite different behavior from that for imaginary chemical potential [56]. $\sigma(\varphi)$ shows a periodicity of $2 \pi$ in $\varphi$, not $2 \pi / 3$ which is required by the RW periodicity. The origin of this difference is the expectation value of the operator $\langle\cdots\rangle_{G}$ in Eq. (33). The subscript $G$ denotes the path integral over the gauge field with a fermion determinant which obeys the ordinary boundary condition. The change of the boundary condition (31) applies only to the Dirac operator. In the case of the imaginary chemical potential, different $\theta$ yields a different fermion determinant, while in the case of the twisted angle the background field does not change with the boundary condition. Therefore, the $\varphi$ dependence of the chiral condensate differ from $\theta$ dependence. In the PNJL model, the authors of Ref. [57] use the value of the Polyakov loop calculated at $\theta=0$ to obtain the chiral condensate $\sigma(\varphi)$. This prescription corresponds to varying only the fermionic boundary condition without changing gluonic background. We will follow the same prescription below. Since the periodicity $2 \pi / 3$ in the imaginary chemical potential is preserved by the RW transition, which is an effect of the Polyakov loop in the context of PNJL model, the relation (34) holds for the normal NJL model calculation which does not couple to $Z(3)$ field. In spite of the absence of confinement in the NJL model, one sees that behavior of the dual chiral condensate is quite similar to the one obtained from lattice QCD and the PNJL model [58].

Figure 12 shows the chiral condensate as a function of the twisted angle $\varphi$, obtained by the same method as used in Ref. [57]. The periodicity is no longer $2 \pi / 3$. One also notes that there is broken phase in the region far from $\varphi=\pi$, even at high temperatures. This is in contrast to the case of the imaginary chemical potential shown in Fig. 6 but similar to what was expected from the gap

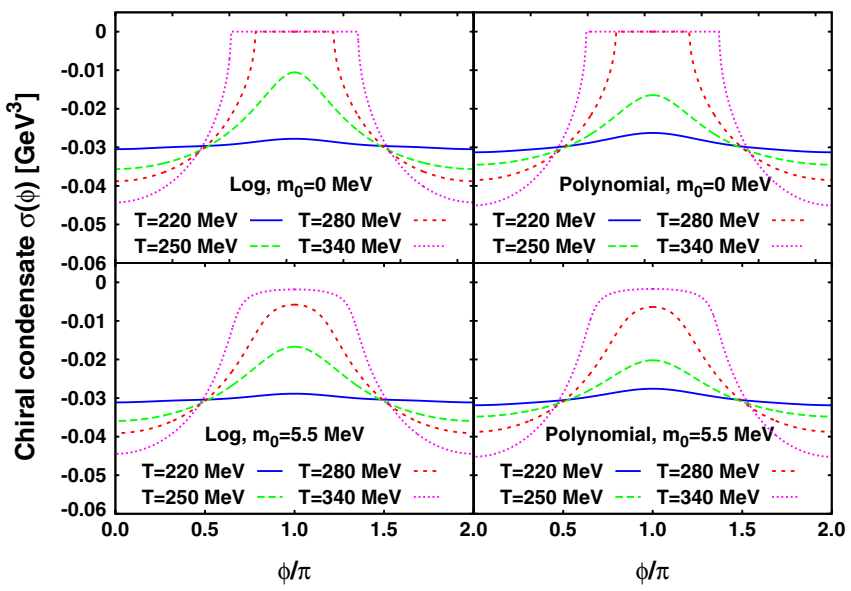

FIG. 12 (color online). Chiral condensate $\sigma=\langle\bar{q} q\rangle$ as a function of the twisted angle $\varphi$. equation of the NJL model, Eq. (28). Indeed, at $\varphi=\pi / 2$ and $3 \pi / 2$, which correspond to $\theta=\mp \pi / 2$, the thermal term vanishes in Eq. (28), resulting in the almost temperature-independent chiral condensate. On the other hand, there are no qualitative differences between the logarithmic potential and the polynomial one. The reason is that the Polyakov loop enters in the gap equation only as a constant determined at $\varphi=\pi$ at each temperature.

Let us introduce a new dual parameter by using $\theta$ instead of $\varphi$ such that it captures the characteristics in the $\theta$ space. We define

$$
\Sigma_{\theta}^{(n)}(T) \equiv \frac{3}{2 \pi} \int_{-\pi / 3}^{\pi / 3} d \theta e^{-i n \theta} \sigma(T, \theta)
$$

The integration region is changed to $[-\pi / 3: \pi / 3]$ owing to the periodicity $2 \pi / 3$. As discussed in Sec. III B, the physical meaning of the periodicity is different in the confinement and deconfinement phases. In the confinement phase, the periodicity $2 \pi / 3$ is due to the term $\cos 3 \theta$ which characterizes the confinement of the quarks. On the other hand, the deconfinement phase is characterized by $\cos \theta$ with a discontinuity at $\theta=\pi / 3+2 \pi k / 3$ caused by the $Z(3)$ transition which thus preserves the periodicity $2 \pi / 3$. Therefore, we expect that $\Sigma_{\theta}^{(1)}$ and $\Sigma_{\theta}^{(3)}$ exhibit a characteristic behavior at the confinement-deconfinement transition.

In Figs. 13 and 14, we compare the three parameters of the confinement-deconfinement transition and their

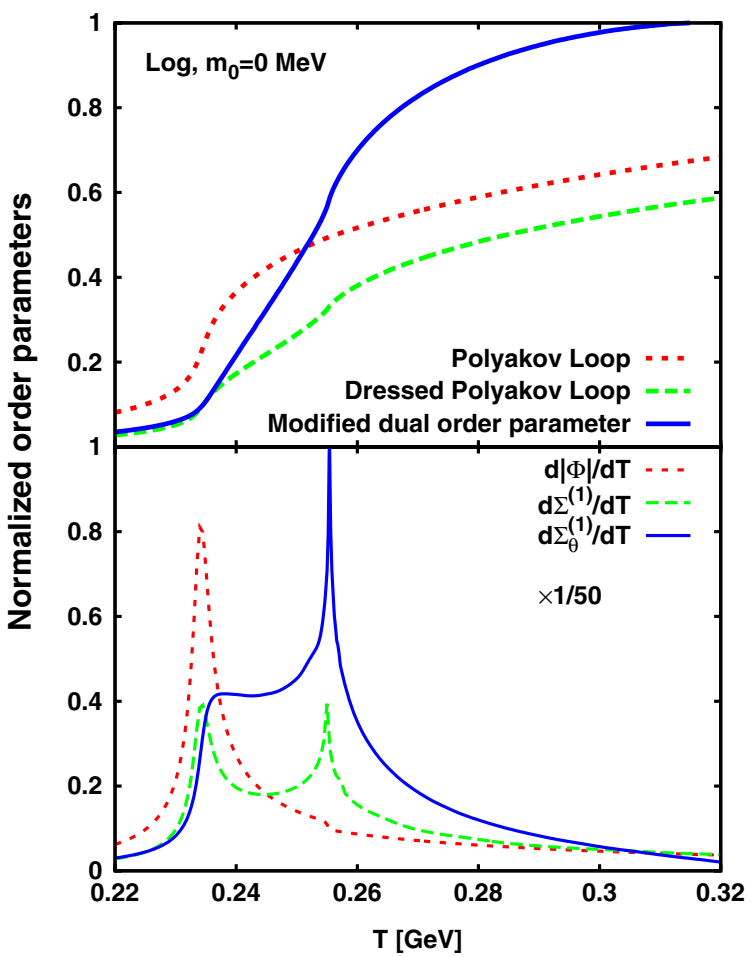

FIG. 13 (color online). Characteristic parameters (upper) and their derivatives with respect to temperature (lower) for the confinement-deconfinement transition in the chiral limit for the logarithmic potential. 


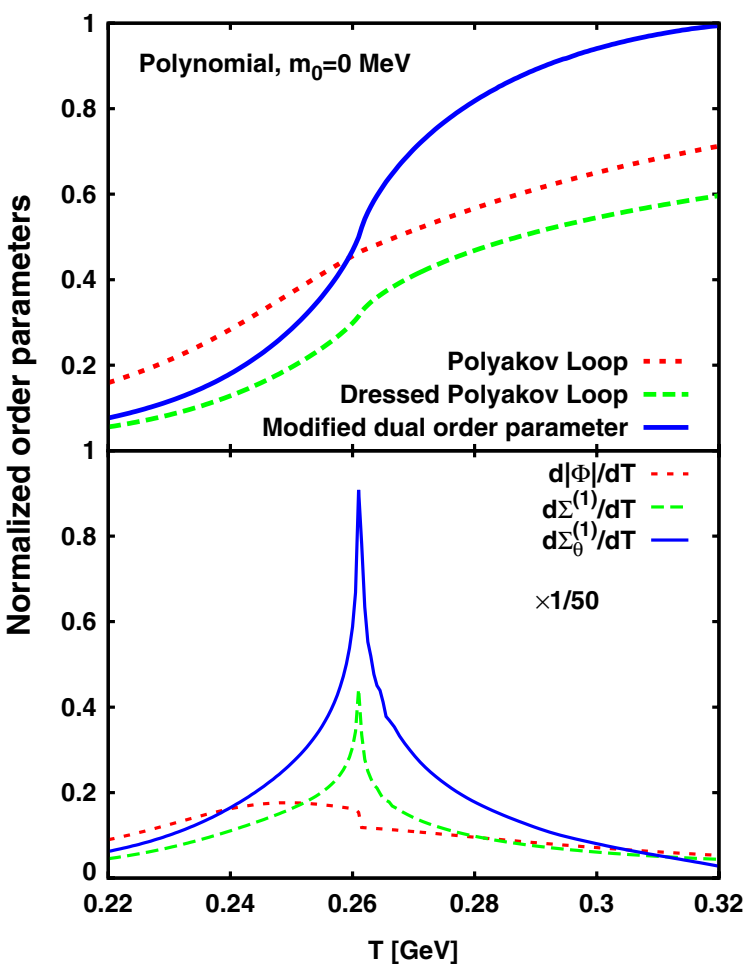

FIG. 14 (color online). Same as Fig. 13, but for the polynomial potential.

derivatives with respect to temperature as functions of temperature. We normalized the dressed Polyakov loop and the modified dual parameter (35) so that they tend to 0 at low temperature and to unity at high temperature by $\Sigma_{\text {norm }}(T) \equiv\left[\Sigma(T)-\Sigma\left(T_{1}\right)\right] /\left[\Sigma\left(T_{2}\right)-\Sigma\left(T_{1}\right)\right]$ where we used $T_{1}=50 \mathrm{MeV}$ and $T_{2}=1 \mathrm{GeV}^{3}$ The same normalization is applied to the derivatives. Note that $\Sigma_{\theta}^{(n)}$ before normalization vanishes at temperature higher than the chiral transition temperature at $\theta=\pi / 3$ in the chiral limit since $\sigma(T, \theta)$ does so, as seen in Fig. 6. After the normalization, it smoothly approaches unity as one sees in Figs. 13-16.

One observes that both dual parameters exhibit a behavior similar to the Polyakov loop. The dressed Polyakov loop is almost parallel to the Polyakov loop in the temperature region covered in the figure. On the other hand, the modified dual parameter $\Sigma_{\theta}^{(1)}$ approaches quickly its limiting value. The behavior around the transition temperatures reflect the difference between the two potentials. In the case of the logarithmic potential, one sees different structures for each derivative of the order parameters. While the derivative of the Polyakov loop $d|\Phi| / d T$ shows only one peak corresponding to the pseudocritical temperature, the dressed Polyakov loop $d \Sigma^{1} / d T$ exhibits a two-peak structure. One agrees with that of $d \Phi / d T$ and the other corresponds to the

\footnotetext{
${ }^{3}$ Note that $\Sigma_{\theta}^{(1)}(T=0)$ does not vanish since the integration is from $-\pi / 3$ to $\pi / 3$, not from $-\pi$ to $\pi$.
}

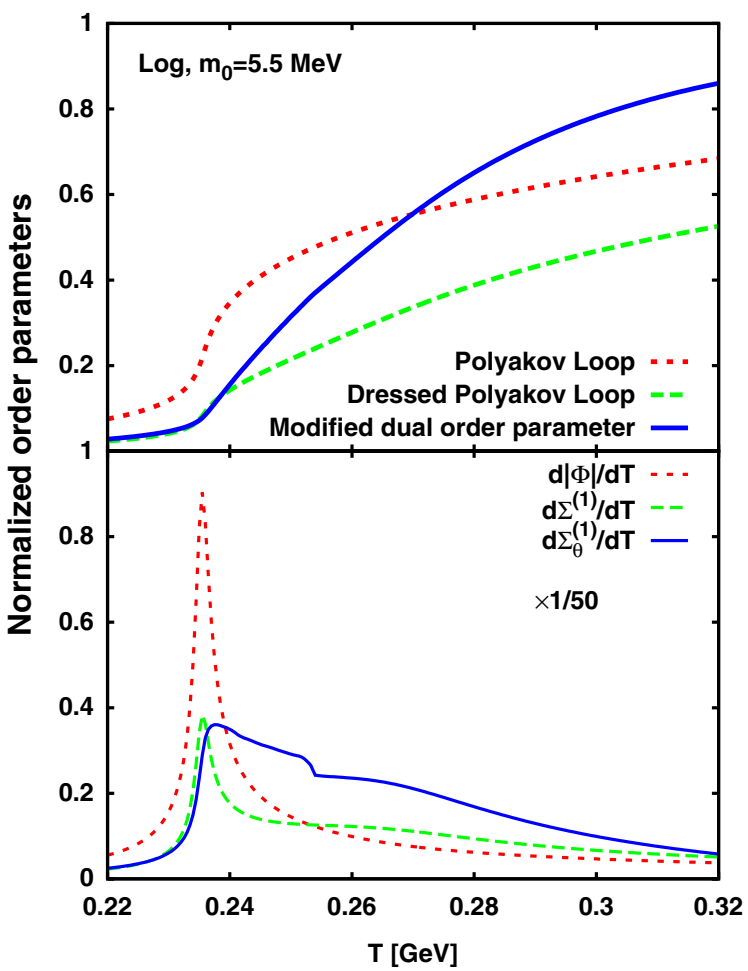

FIG. 15 (color online). Same as Fig. 13, but for $m_{0}=5.5 \mathrm{MeV}$.

chiral transition (see Fig. 2). The derivative of the modified dual parameter $d \Sigma_{\theta}^{(1)} / d T$ also shows a peak for the chiral transition. However, the deconfinement transition appears only as a shoulder. The polynomial potential shows a broader peak in $d \Phi / d T$ reflecting the weaker nature of transition. However, maxima in the dual order parameters associated with the crossover transition do not appear.

Inclusion of a small quark mass, $m_{0}=5.5 \mathrm{MeV}$ slightly modifies the behavior of the characteristic parameters, as expected from the difference in $\sigma$. Figs. 15 and 16 show the three parameters and their derivatives for $m_{0}=5.5 \mathrm{MeV}$. While at finite quark mass there is little difference in the behavior of the parameters compared to the $m_{0}=0$ case, distinct peak structures show up in their derivatives. For the logarithmic potential, the peak associated with the chiral transition seen in the chiral limit does not exist in either of the dual parameters. The remnant of the chiral transition appears only in the modified dual parameter as a dip. On the other hand, polynomial potential exhibits much broader peaks, which correspond to deconfinement in $d|\Phi| / d T$ and to the chiral transition in the dual parameters.

Figure 17 shows the dual parameter (32) and (35) for $n=3$. In all the cases, they start by growing, then exhibit a peak structure and then decrease. This is common for both $\Sigma^{(3)}$ and $\Sigma_{\theta}^{(3)}$. This behavior of $\Sigma_{\theta}^{(3)}$ can be understood by analyzing $\sigma(T, \theta)$, which is shown in Fig. 6. At low $T$, it oscillates according to $\cos 3 \theta$ with an amplitude given 


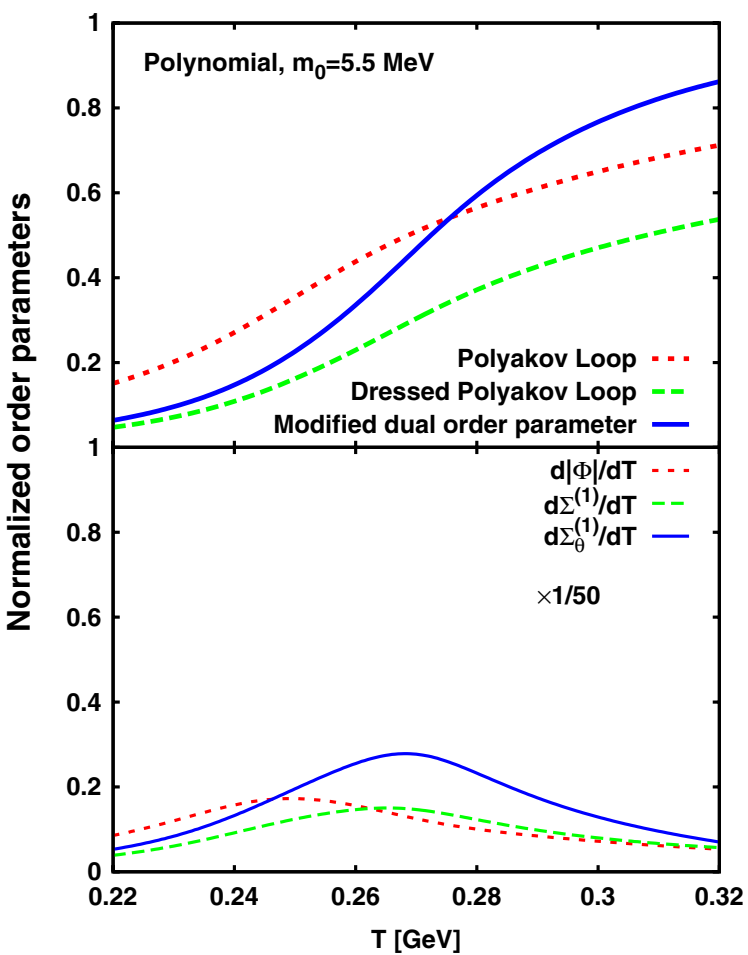

FIG. 16 (color online). Same as Fig. 14, but for $m_{0}=5.5 \mathrm{MeV}$.

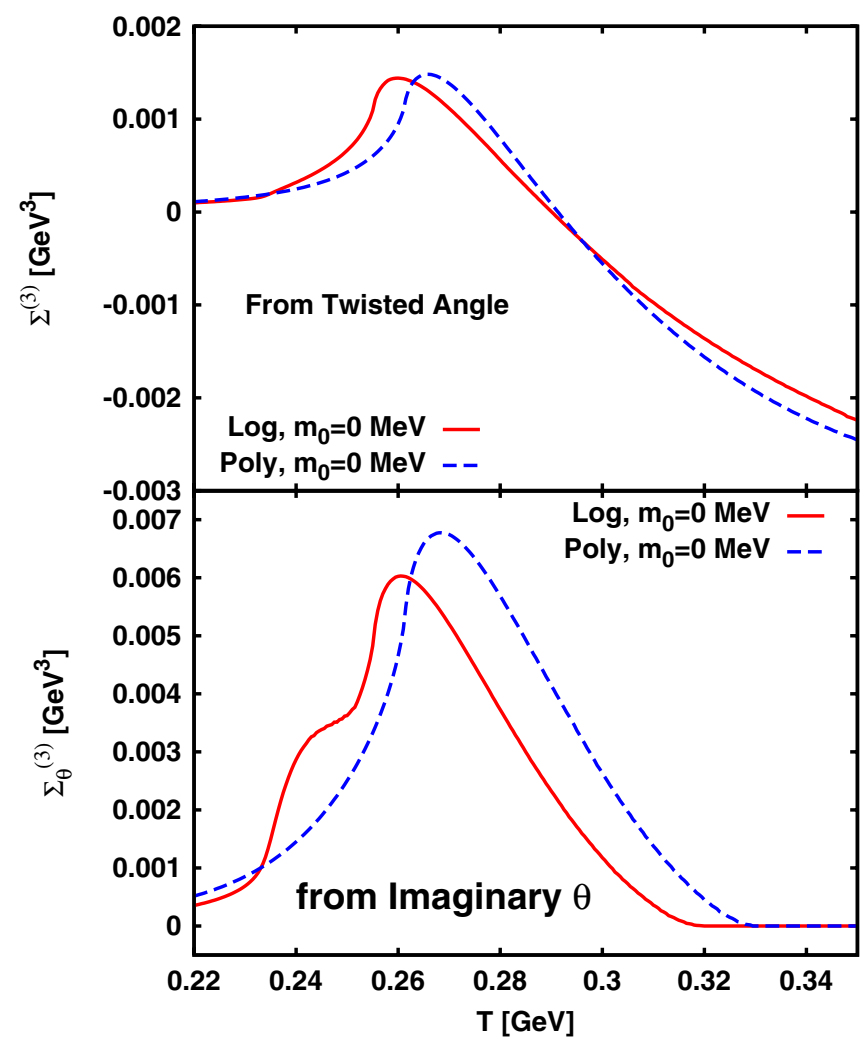

FIG. 17 (color online). Dual order parameters of $n=3$. The upper panel shows the result from Eq. (32) while lower one displays that from Eq. (35). by the thermal factor. Then $\Sigma_{\theta}^{(3)}$, which is proportional to the amplitude, increases with $T$. At $T>T_{\chi}, \sigma(\theta, T)$ vanishes in the interval $\theta=\left[0: \theta_{c}\right]$ thus only the region $\left[\theta_{c}: \pi / 3\right]$ contributes to the integral. Therefore, $\Sigma_{\theta}^{(3)}$ has a peak at $T=T_{\chi}$ and then decreases. This is not related to the deconfinement phenomenon and is common for $\Sigma^{(3)}$ and $\Sigma_{\theta}^{(3)}$.

If one focuses on the difference between the logarithmic and the polynomial potential, however, one finds a remnant of the deconfinement-confinement transition in the behavior of $\Sigma_{\theta}^{(3)}$. As we have seen in Fig. 7, $\sigma(T, \theta)$ has a discontinuity induced by the first-order confinement-deconfinement transition. It is reflected to the nonmonotonic behavior of $\Sigma_{\theta}^{(3)}$ between $T=0.24$ and $T=0.26 \mathrm{GeV}$ in the case of the logarithmic potential. Indeed, the shoulder seen at $T=0.24 \mathrm{GeV}$ corresponds to the temperature of the CEP, at which $\sigma(\theta, T)$ has a discontinuity, and the second inflection point reflects the RW end point which is the triple point in this case. This indicates that the newly introduced dual parameter $\Sigma_{\theta}^{(3)}$ is sensitive to the confinement-deconfinement transition at imaginary chemical potential.

\section{SUMMARY}

We have studied the confinement-deconfinement transition in the PNJL model at imaginary chemical potential with the simplest interaction. We discussed the origin of the characteristic periodicity $2 \pi / 3$ of the order parameters. It is characterized by $\cos 3 \theta$ in the confined phase while it is due to $\cos \theta$ with the RW transition at $\theta=\pi / 3+2 \pi k / 3$ induced by the change of the phase of the Polyakov loop. We also explored the results from different Polyakov-loop potentials. We found that the property of the confinementdeconfinement transition depends on the choice of the potential in spite of the fact that both potentials exhibit the first-order phase transition in the absence of quarks. Substantial differences are seen in both the RW end point and the behavior of the Polyakov loop at finite $\theta$. In the case of the logarithmic potential, we find that the confinement-deconfinement transition becomes first-order near $\theta=\pi / 3$ and there is a critical end point of the transition at imaginary chemical potential. We also find that the location of the CEP moves with the four fermion coupling $G_{s}$ and it reaches real value of the chemical potential by increasing $G_{s}$. This behavior can be understood by the suppression of the quark contribution since increasing $G_{s}$ implies larger $\langle\bar{q} q\rangle$ which quantifies the dynamical quark mass. At large coupling, the existence of the CEP is independent of the choice of the potential. However, the polynomial potential requires a larger $G_{s}$ because it exhibits a much weaker first-order transition. Consequently, it seems that the order of the deconfinement transition is determined by the size of the gap $\Delta \Phi$ in the Polyakov-loop potential and the quark condensate $\langle\bar{q} q\rangle$. 
The first-order phase transition influences the behavior of the chiral condensate as a sudden jump at the critical imaginary chemical potential. We proposed a modified dual parameters using the imaginary chemical potential based on the analogy to the twisted angle in the dual order parameters. Comparing the $n=1$ case with the Polyakov loop and the dressed Polyakov loop, we found that each parameter has different sensitivity to the phase transitions. We showed that the $n=3$ case has a characteristic behavior owing to the first-order confinement-deconfinement transition at intermediate $\theta$. We expect that our study is relevant for understanding the QCD phase diagram.

\section{ACKNOWLEDGMENTS}

K. M. would like to thank Y. Sakai, T. Sasaki, and M. Yahiro for fruitful discussion and warm hospitality during his visit to Kyushu University. K.M. and V.S. would like to acknowledge Frankfurt Institute of Advanced Study (FIAS) for support. K. M. is supported by the Yukawa International Program for Quark Hadron Sciences at Kyoto University. B.F. and K. R. acknowledges partial support by EMMI. K. R. acknowledges partial support by the Polish Ministry of Science (MEN).
[1] A. Bazavov et al., Phys. Rev. D 80, 014504 (2009).

[2] Y. Aoki, S. Borśanyi, S. Dürr, Z. Fodor, S. D. Katz, S. Krieg, and K. Szabo, J. High Energy Phys. 06 (2009) 088.

[3] Y. Aoki, G. Endrödi, Z. Fodor, S. D. Katz, and K. K. Szabo, Nature (London) 443, 675 (2006).

[4] S. Muroya, A. Nakamura, C. Nonaka, and T. Takaishi, Prog. Theor. Phys. 110, 615 (2003).

[5] A. Roberge and N. Weiss, Nucl. Phys. B275, 734 (1986).

[6] S. Ejiri, Phys. Rev. D 78, 074507 (2008).

[7] A. Li, A. Alexandru, K. F. Liu, and X. Meng, Phys. Rev. D 82, 054502 (2010).

[8] P. de Forcrand and O. Philipsen, Nucl. Phys. B642, 290 (2002).

[9] P. de Forcrand and O. Philipsen, Nucl. Phys. B673, 170 (2003).

[10] M. D'Elia and M. P. Lombardo, Phys. Rev. D 67, 014505 (2003).

[11] P. Giudice and A. Papa, Phys. Rev. D 69, 094509 (2004); P. Cea, L. Cosmai, M. D’Elia, C. Manneschi, and A. Papa, ibid. 80, 034501 (2009).

[12] M. D'Elia and M. P. Lombardo, Phys. Rev. D 70, 074509 (2004).

[13] H.S. Chen and X. Q. Luo, Phys. Rev. D 72, 034504 (2005).

[14] M. D’Elia, F. D. Renzo, and M. P. Lombardo, Phys. Rev. D 76, 114509 (2007).

[15] P. de Forcrand and O. Philipsen, J. High Energy Phys. 01 (2007) 077.

[16] L. K. Wu, X. Q. Luo, and H. S. Chen, Phys. Rev. D 76, 034505 (2007).

[17] P. de Forcrand and O. Philipsen, Phys. Rev. Lett. 105, 152001 (2010).

[18] K. Nagata and A. Nakamura, Phys. Rev. D 83, 114507 (2011).

[19] A. Hart, M. Laine, and O. Philipsen, Phys. Lett. B 505, 141 (2001).

[20] M. Bluhm and B. Kämpfer, Phys. Rev. D 77, 034004 (2008).

[21] N. Weiss, Phys. Rev. D 35, 2495 (1987).

[22] H. Kouno, Y. Sakai, K. Kashiwa, and M. Yahiro, J. Phys. G 36, 115010 (2009).
[23] M. D'Elia and F. Sanfilippo, Phys. Rev. D 80, 111501(R) (2009).

[24] Y. Sakai, T. Sasaki, H. Kouno, and M. Yahiro, Phys. Rev. D 82, 076003 (2010).

[25] C. Bonati, G. Cossu, M. D'Elia, and F. Sanfilippo, Phys. Rev. D 83, 054505 (2011).

[26] G. Aarts, S. P. Kumar, and J. Rafferty, J. High Energy Phys. 07 (2010) 056.

[27] K. Fukushima, Phys. Lett. B 591, 277 (2004).

[28] C. Ratti, M. A. Thaler, and W. Weise, Phys. Rev. D 73, 014019 (2006).

[29] Y. Nambu and G. Jona-Lasinio, Phys. Rev. 122, 345 (1961).

[30] Y. Nambu and G. Jona-Lasinio, Phys. Rev. 124, 246 (1961).

[31] T. Hatsuda and T. Kunihiro, Phys. Rep. 247, 221 (1994).

[32] H. T. Elze, D. E. Miller, and K. Redlich, Phys. Rev. D 35, 748 (1987).

[33] D. E. Miller and K. Redlich, Phys. Rev. D 37, 3716 (1988).

[34] Y. Sakai, K. Kashiwa, H. Kouno, and M. Yahiro, Phys. Rev. D 77, 051901(R) (2008).

[35] Y. Sakai, K. Kashiwa, H. Kouno, and M. Yahiro, Phys. Rev. D 78, 036001 (2008).

[36] Y. Sakai, K. Kashiwa, H. Kouno, M. Matsuzaki, and M. Yahiro, Phys. Rev. D 78, 076007 (2008).

[37] Y. Sakai, K. Kashiwa, H. Kouno, M. Matsuzaki, and M. Yahiro, Phys. Rev. D 79, 096001 (2009).

[38] Y. Sakai, H. Kouno, and M. Yahiro, J. Phys. G 37, 105007 (2010).

[39] K. Kashiwa, T. Hell, and W. Weise, Phys. Rev. D 84, 056010 (2011).

[40] E. Bilgici, F. Bruckmann, C. Gattringer, and C. Hagen, Phys. Rev. D 77, 094007 (2008).

[41] C. Sasaki, B. Friman, and K. Redlich, Phys. Rev. D 75, 074013 (2007).

[42] R. D. Pisarski, Phys. Rev. D 62, 111501(R) (2000).

[43] G. Boyd, J. Engles, F. Karsch, E. Laermann, C. Legeland, M. Lütgemeier, and B. Petersson, Nucl. Phys. B469, 419 (1996).

[44] O. Kaczmarek, F. Karsch, P. Petreczky, and F. Zantow, Phys. Lett. B 543, 41 (2002). 
[45] S. Roessner, C. Ratti, and W. Weise, Phys. Rev. D 75, 034007 (2007).

[46] K. Fukushima, Phys. Rev. D 77, 114028 (2008).

[47] C. Sasaki and I. Mishustin, Phys. Rev. C 82, 035204 (2010).

[48] C. Wozar, T. Kaestner, A. Wipf, T. Heinzl, and B. Pozsgay, Phys. Rev. D 74, 114501 (2006).

[49] K. I. Kondo, Phys. Rev. D 82, 065024 (2010).

[50] J. Braun, L. M. Haas, F. Marhauser, and J. M. Pawlowski, Phys. Rev. Lett. 106, 022002 (2011).

[51] E. S. Bowman and J. I. Kapusta, Phys. Rev. C 79, 015202 (2009).

[52] V. Skokov, B. Friman, E. Nakano, K. Redlich, and B.-J. Schaefer, Phys. Rev. D 82, 034029 (2010).
[53] L. McLerran, K. Redlich, and C. Sasaki, Nucl. Phys. 824, 86 (2009).

[54] L. McLerran and R. D. Pisarski, Nucl. Phys. 796, 83 (2007).

[55] C. S. Fischer and J. A. Mueller, Phys. Rev. D 80, 074029 (2009).

[56] E. Bilgici, J. D. F. Bruckmann, C. Gattringer, C. Hagen, E. M. Ilgenfritz, and A. Maas, Few-Body Syst. 47, 125 (2010).

[57] K. Kashiwa, H. Kouno, and M. Yahiro, Phys. Rev. D 80, 117901 (2009).

[58] T. K. Mukherjee, H. Chen, and M. Huang, Phys. Rev. D 82, 034105 (2010). 